\title{
The Contingent Smile: A Meta-Analysis of Sex Differences in Smiling
}

\author{
Marianne LaFrance \\ Yale University
}

\author{
Marvin A. Hecht \\ Oracle Corporation
}

\author{
Elizabeth Levy Paluck \\ Yale University
}

\begin{abstract}
The authors present a meta-analysis of sex differences in smiling based on 448 effect sizes derived from 162 research reports. There was a statistically significant tendency for women and adolescent girls to smile more than men and adolescent boys $(d=0.41)$. The authors hypothesized that sex differences in smiling would be larger when concerns about gender-appropriate behavior were made more conspicuous, situational constraints were absent or ambiguous, or emotion (especially negative) was salient. It was also predicted that the size of the sex difference in smiling would vary by culture and age. Moderator analysis supported these predictions. Although men tend to smile less than women, the degree to which this is so is contingent on rules and roles.
\end{abstract}

It is virtually a cliché of Western culture that women are both more emotional and more expressive than men. Although the extent of sex differences ${ }^{1}$ in measured emotionality remains in dispute (Brody, 1997; Fischer, 1993; LaFrance \& Banaji, 1992), there is substantial agreement that women are more overtly expressive than men (Briton \& Hall, 1995a; Brody, 1999; J. A. Hall, 1984; Hess et al., 2000; Kring \& Gordon, 1998; Manstead, 1991). Some studies have shown that women are more expressive even when angry, although the degree to which the sexes differ in how much they express anger is very context and measurement specific (Archer, 2000; Fischer, 2002; Kring, 2000). With one facial expression however, there is near unanimity-women smile more than men (Briton \& Hall, 1995a; J. A. Hall \& Halberstadt, 1986; LaFrance \& Hecht, 1999). The circumstances associated with this difference are the subject matter of this report.

Human smiling would appear to be an uncomplicated facial expression with a straightforward meaning. Yet, smiling turns out to be one of the most complex facial displays. For one thing, smiles can take a variety of forms (Ekman \& Friesen, 1982), and for another, they are found in an improbable variety of situations. In fact, the novelist Herman Melville (1852/1949) maintained that

Marianne LaFrance and Elizabeth Levy Paluck, Department of Psychology, Yale University; Marvin A. Hecht, Oracle Corporation, Redwood Shores, California.

Preparation of this article was supported by doctoral and postdoctoral grants from the Social Sciences and Humanities Research Council of Canada and Grant NSF SBR-9319897 from the National Science Foundation. We thank Alison Noyes, Larry DeSalvatore, Susan Rivers, Keith Lyle, Steven Tallides, and Leathe Allard for their assistance with coding. We also appreciate the statistical advice offered by Alice Eagly, Blair Johnson, and Robert Rosenthal.

Correspondence concerning this article should be addressed to Marianne LaFrance, Department of Psychology, Yale University, P.O. Box 208205, New Haven, Connecticut. E-mail: marianne.lafrance@yale.edu; marvin .hecht@oracle.com; or elizabeth.paluck@yale.edu "a smile is the chosen vehicle for all ambiguities" (p. 98). The ambiguity may be at the heart of the difficulty in accounting for why women tend to smile more than men, or alternatively, why men tend to smile less than women.

For more than 100 years, natural and social scientists have probed the many meanings associated with the human smile (e.g., Darwin, 1872/1965). Although smiles reflect positive affect (Ekman, Davidson, \& Friesen, 1990; Ekman \& Friesen, 1982; Ekman, Friesen, \& Ancoli, 1980), people also smile when the relevant affect is anything but positive. People smile when they are embarrassed (Edelmann, Asendorpf, Contarello, \& Zammuner, 1989), uncomfortable (Ochanomizu, 1991), miserable (Ekman \& Friesen, 1982), and socially apprehensive (Ickes, Patterson, Rajecki, \& Tanford, 1982).

Indeed, it has been suggested that smiling is less a sign of underlying emotion than a social display meant for others. (See Ekman, 1994, for a critique of the idea that expressions are more social tools than readouts of emotion states.) Nonetheless, Fridlund and his colleagues found that people smiled more in the imagined presence of someone they knew than when alone, even when there were no differences in how happy they were (Fridlund, 1994; Fridlund et al., 1990). Similarly, Kraut and Johnston (1979) found that bowlers who had just been successful smiled more when they turned to face their friends than immediately following their accomplishment.

Social motives have also been associated with the occurrence of smiling. Greetings are often coupled with smiling (Eibl-Eibesfeldt, 1989), as are persuasion attempts aimed at patients (Burgener, Jirovec, Murrell, \& Barton, 1992), voters (Masters, Sullivan, Lanzetta, McHugo, \& Englis, 1985; Mullen et al., 1986), students (Zanolli, Saudargas, \& Twardosz, 1990), and potential dates

\footnotetext{
${ }^{1}$ In this article, we use the term sex differences in the context of comparing the two sexes. The term gender is most often used in the context of societal norms and roles associated with each sex.
} 
(Walsh \& Hewitt, 1985). Smiles have also been shown to elicit greater leniency for transgressors (LaFrance \& Hecht, 1995) and to ward off others' displeasure (Elman, Schulte, \& Bukoff, 1977; Goldenthal, Johnston, \& Kraut, 1981).

Smiling is even a social requirement on occasion. For example, Wierzbicka (1994) observed that cheerfulness is mandatory in many cultures. Within the United States, Hochschild (1983) noted that some workers are required to smile as part of their jobs. For instance, airline flight attendants must smile and smile well. Thus, a flight attendant is trained to "really work on her smiles" and is expected to "manage her heart" in such a way as to create a smile that seems both "spontaneous and sincere" (Hochschild, 1983, p. 105; see also E. J. Hall, 1993). In short, smiling is a socially contingent facial expression, and any attempt to understand sex differences in smiling needs to take such contingencies into account.

In this review, we examine several contingencies that impact when sex differences in smiling are to be expected and when they are not (LaFrance \& Hecht, 1999). First, we contend that there are gender display rules that call for women to smile more than men (at least in Western cultures). These gender display rules are most in evidence when people are aware of being noticed or evaluated. Second, we look at situational contingencies, and our prediction was that women and men would display similar rates of smiling when they occupied the same social roles or were engaged in the same social tasks. Third, we explore the idea that larger sex differences in smiling are observed when circumstances are emotionally charged (especially negatively) because such situations call on women to do the "emotion work" that such contexts require. Hochschild (1983) introduced the term emotion work to describe efforts to create and maintain positive feelings and alleviate and rectify negative ones in self or in others-smiling would seem to be a particularly effective way to achieve these outcomes. Finally, we discuss our expectation that, because facial expressions are also influenced by cultural display rules, sex differences in smiling would be variously manifest depending on participants' cultural affiliation.

\section{Sex Differences in Smiling}

Research on nonverbal behavior confirms that women are both better encoders and decoders of emotion (J. A. Hall, 1984; Manstead, 1991). In fact, three expressive domains - namely, smiling, general expressiveness, and decoding accuracy_have been well examined and show relatively large sex differences (J. A. Hall, Carter, \& Horgan, 2000). In particular, one of the largest and most reliable findings of behavior research is that men smile less than women (J. A. Hall, 1984; J. A. Hall \& Halberstadt, 1986; Henley, 1977). Nonetheless, there is considerable debate as to what factors account for this difference. In what follows, we briefly review several hypotheses advanced by J. A. Hall and others in metaanalyses of sex differences in smiling (J. A. Hall, 1984; J. A. Hall $\&$ Halberstadt, 1986). We revisit some of their conclusions as well as present a number of new hypotheses in light of what is now a substantially larger body of empirical findings.

\section{Prior Meta-Analyses of Sex Differences in Smiling}

In 1984, J. A. Hall published the first meta-analysis on sex differences in smiling, which showed women smiled more than men. Based on 20 published studies, the effect size $(d)$ she reported was a moderate 0.63 . In a follow-up report, J. A. Hall and Halberstadt (1986) added seven new cases and reported a somewhat lower weighted effect size of 0.42 . In the latter report, three moderators were considered to moderate the size of observed sex differences in smiling.

\begin{abstract}
The warmth-affiliation hypothesis suggests that women smile and gaze more than men do because of their socioemotional orientation. ... The dominance-status hypothesis suggests that women smile more than men do because they are socially weaker... [and the] social tension-nervousness hypothesis... suggests that women's greater smiling reflects attempts to mask or cope with social unease. (J. A. Hall \& Halberstadt, 1986, pp. 138-139)
\end{abstract}

In their meta-analysis, studies were coded to assess the degree to which various moderators were associated with the observed sex difference in smiling. Level of acquaintanceship was a moderator to test the warmth-affiliation hypothesis; status was a moderator for the dominance-status hypothesis, and comfortableness was the moderator for the social-tension hypothesis. Each moderator was correlated with the size of the sex difference as well as analyzed with a series of multiple regressions. These analyses led J. A. Hall and Halberstadt (1986) to conclude that the social-tension hypothesis (three moderators) received "strong support," that the warmthaffiliation hypothesis (three moderators) received "weak" support (p. 153), and that the dominance-status hypothesis (one moderator) was not supported by the data. Several explanations for the link between social tension and sex differences in smiling were considered, including the possibility that smiling in situations of high social tension represents an attempt to be cheerful in spite of the tense situation or an effort to ease the other person's discomfort.

\section{Aims for the Present Meta-Analysis}

The present meta-analysis takes another look at the literature examining the relationship between gender and smiling. The reasons for this second look are several. First, it is occasioned by the availability of what is now a substantially larger set of relevant research reports. Sex differences in smiling have been measured in a large number of studies published since the J. A. Hall and Halberstadt (1986) analysis, even when that particular relationship was not the central question for the research. We included in our meta-analysis unpublished studies such as conference papers and theses, as well as previously unanalyzed data that were not included in their prior meta-analysis.

Second, we explored the influence of several moderators derived from work in other areas of sex difference research. Theory suggests, for instance, that the sexes tend to present themselves in more or less gender-stereotypic ways in response to environment cues that make gender salient (Deaux \& Major, 1987). For example, in classic studies by Zanna and his colleagues (Von Baeyer, Sherk, \& Zanna, 1981; Zanna \& Pack, 1975), women exhibited more or less stereotypically feminine behaviors depending on how they thought a male interviewer or potential date viewed women. Even incidental exposure to gender-relevant cues such as advertisements in fashion magazines has been shown to increase gender self-stereotyping (Chiu et al., 1998). Such effects occur because by the time individuals reach their teen years, they are well versed in what constitutes expected gender-normative behavior. Conse- 
quently, we contend that norms for gender-appropriate expression-in particular, that of calling for women to smile more than men-are activated when participants believe that their behavior is being monitored or evaluated in some way.

In addition, there was reason to consider the possibility that the size of the sex difference in smiling might vary across cultural groups. Although little research has addressed the joint impact of culture and gender on expressive behavior, findings indicate that sex differences in expressiveness are larger in some cultures than in others. Joshi and Maclean (1994) reported that Indian preschool girls were more inclined to report facial masking of negative emotion than either Indian preschool boys or British preschoolers of either sex, a finding they attributed to the greater emphasis on decorum and deference to adults in the socialization of Indian girls. Among adults, Frymier, Klopf, and Ishii (1990) compared American and Japanese responses on an affect scale. They noted no sex differences for Japanese participants but substantial sex differences for the American sample. Although much of the available research on sex differences in smiling has focused on Western cultures, a large database of studies on gender and smiling allows us to determine whether there are nationality differences and ethnic differences within the United States.

The third goal for the present meta-analysis was to conduct a more fine-grained analysis of several moderators previously considered by J. A. Hall and Halberstadt (1986) but which could not be fully tested at the time because of a relatively modest database. For example, like J. A. Hall and Halberstadt, we examined the impact of tension and nervousness on the size of the sex difference in smiling, but we considered the possibility that there are different kinds of tension. Specifically, we distinguished between social tension, as might occur when an interpersonal interaction is strained in some respect, and task tension, which could be precipitated by anticipated evaluation of one's performance. In addition, our larger database permitted a more complete test of a controversial moderator of sex differences in smiling, namely, status or power differences. As noted above, J. A. Hall and Halberstadt concluded that the dominance-status moderator was uncorrelated with the size of the sex difference. However, their sample did not include studies in which participants could be coded as occupying a low-status position. In the present review, we were able to locate 69 cases that could be coded for low levels of social power. We turn now to a discussion of the moderators we propose for the present meta-analysis and our theoretical rationale for their inclusion.

\section{Theoretical Perspectives on Sex Differences in Smiling}

This article begins with the premise that smiling is a significant social behavior that shows a reliable sex difference. Drawing on several "wide-lens" theories of sex differences as well as recent research in the area of nonverbal communication, we propose that the degree to which sex differences in smiling are found depend on social surroundings and social standards. Specifically, we propose three core contextual factors are key to understanding sex differences in smiling (LaFrance \& Hecht, 1999). These are genderbased norms, situational constraints, and emotion salience.

\section{Gender-Based Norms for Smiling}

A standard finding in the literature on social roles and norms is that when a relevant role or norm is made salient, individuals respond in role- or norm-congruent ways (Cialdini, Reno, \& Kallgren, 1990; Turner, Hogg, Oakes, Reicher, \& Wetherell, 1987). In particular, there is agreement that the behavior of men and women is often guided by ideas about what is and is not gender-normative behavior. According to Deaux and Major's (1987) gender-incontext theory, people behave in accord with "gender belief systems," which are activated when something about a particular situation makes gender salient. Within the more specific nonverbal communication literature, Ekman and Friesen (1975) coined the term display rules to refer to the set of guidelines learned by individuals through socialization that dictate the socially appropriate management of facial expression. Display rules might specify that a particular facial expression be intensified, deintensified, neutralized, or masked with another emotion.

Although several researchers have focused on articulating cultural display rules and specifically at what point in development they become part of a person's repertoire (e.g., Ekman \& Friesen, 1975; Saarni, 1993), gender-based display rules have been given less research attention. Nonetheless, several lines of research indicate the presence of norms that call for females to smile more than males. For example, Cole (1985) found that among children, girls were more likely than boys to display positive expressions upon receiving a disappointing gift. Among adults, men say that they smile less than women (Korzenny, Korzenny, \& Sanchez de Rota, 1985), and both sexes believe that to be the case (Briton \& Hall, 1995a, 1995b; Kramer, 1977). Studies also show that nonsmiling women are judged as experiencing more negative affect than nonsmiling men (Deutsch, LeBaron, \& Fryer, 1987) and that smiling males are perceived to be less effective than nonsmiling males (Kierstead, D'Agostino, \& Dill, 1988). In addition, women more than men anticipate that others will think less well of them if they do not smile in response to a friend's good news (LaFrance, 1997). In sum, not only are women and adolescent girls thought to smile more than men and adolescent boys, they are enjoined to do so. Gender norms for smiling, like gender norms more broadly, likely act both as self-imposed prescriptions (Fiske \& Stevens, 1993) and as self-fulfilling prophecies imposed by others (Fischer, 1993)

Several moderators are proposed to tap the operation of sexbased norms for smiling. They are (a) observation awareness, (b) presence of others, (c) engagement with others, (d) instructions to get acquainted, (e) research setting, (f) archival materials, (g) posing for photographs, and (h) familiarity. These moderators converge on the idea that expressive displays are affected by who is present and what the context is (Fridlund, 1994). Specifically, when people believe their behavior is under scrutiny or when they are with unfamiliar others or when they know they are being evaluated, they take more care to be suitably expressive. And one important component of suitable display involves genderappropriate smiling behavior. For example, Banerjee and Lintern (2000) found that the gender typing displayed by young children is part of an active self-presentational effort to win positive evaluation from peers. Specifically, younger boys presented themselves as more gender-typed in front of a peer audience than when alone. 
Among adults, Kleck et al. (1976) found that men showed less pain-related expressivity when observed than when alone.

We predicted that the sex difference in smiling (with males smiling less) would be greater when participants were aware of being observed, such as when people knew they were being videotaped. Moreover, we predicted that presence of others, more focused engagement with others, and explicit instructions to get acquainted with others would also lead to larger sex differences in smiling because when others are near at hand and focused faceto-face interaction is expected, people adhere more closely to norms for gender-appropriate behavior. Likewise, we expected the research setting to moderate smiling differences because participants in a laboratory in contrast to a field setting probably assume that they are being monitored and evaluated. We predicted that archival materials would generate large sex differences in smiling because visual images are often constructed with the precise goal of presenting idealized gendered images (Goffman, 1979). In other words, advertisements and even casual photos show women and men who have adopted exemplary or model expressions.

The last moderator in this cluster is familiarity with interaction partners. We predicted that familiarity with interaction partners would lessen concern about appropriate behavior and hence produce a smaller sex difference in the amount of smiling shown by participants. Support for this conjecture comes from research on self-presentational concerns in everyday interaction. Leary et al. (1994) found that people showed lower motivation to enhance the views of familiar same-sex others toward themselves than with low-familiar, same-sex others.

Cultural factors. Cultural groups likely vary in the degree to which they accentuate sex differences in expressive behavior. For example, Matsumoto and Ekman (1989) suggested that because perceptions of sex differences in facial expressivity appear to show cross-cultural variability, there might be actual sex differences across cultures. Consequently, nationality (sample primarily consisted of North American and European countries) and ethnicity (within the United States) were coded as moderators.

Developmental factors. J. A. Hall and Halberstadt (1986) reported finding no significant sex difference in smiling for young children, even though they found a sex difference in smiling for adults. Some researchers have proposed that this reflects the influence of modeling and reinforcement whereby people acquire and heed gender norms for smiling (e.g., Manstead, 1991). In this analysis, we focus on adolescents and adults. We predicted that sex differences in smiling would be at their zenith for adolescents and young adults when differences between the sexes were emphasized (Eccles \& Bryan, 1994) but would be smaller for older adults.

Partner demographics. In addition to coding participant demographics (sex, nationality, ethnicity, age), we also coded these attributes for the participants' interaction partners on the grounds that partner attributes constitute an important part of the social context. For example, there may be a smaller sex difference in smiling when the partner is younger or older because in such encounters both male and female participants might respond in a more similar fashion to people who are in different age groups than themselves. A similar logic holds for interactions in which participants' partners are a different sex or different culture than themselves.

\section{Situational Constraints}

The second key idea guiding our meta-analysis was that expressive behavior is constrained and guided by the situations, roles, and tasks that people find themselves responding to. Indeed, considerable psychological research (see Mischel, 1977) has testified to the power of the situation in affecting behavior. In other words, we contend that there are situation-specific display rules that have the same impact as other situational factors described by Mischel (1977):

Psychological "situations" (stimuli, treatment) are powerful to the degree that they lead everyone to construe the particular events in the same way, induce uniform expectancies regarding the most appropriate response pattern, provide adequate incentives for the performance of that response pattern and require skills that everyone has to the same extent. (p. 348)

In the context of gender-related behavior, social role theory makes a similar argument, namely that sex differences emerge when men and women occupy different roles in society. Sex differences are argued to be smaller when women and men occupy similar roles and thus experience similar constraints on and similar expectations for their behavior (Eagly, 1987). A relevant study was carried out by Moskowitz, Suh, and Desaulniers (1994). They examined the behavior of women and men assigned to different work roles. Specifically, they examined dominance behavior when participants were supervisors, coworkers, or people being supervised. They found that assignment to one of these roles significantly influenced the degree to which both sexes showed dominance behavior. Both sexes were more dominant in a supervisory role and more submissive as a supervisee. Moreover, it is worth noting that the Moskowitz et al. data were based on within-subject analyses. In other words, their data showed people shifting from dominant to submissive behaviors as a function of their current role rather than previous role occupancy.

In sum, our predictions for the microlevel behavior of smiling mirror the theoretical premises of social role theory, the latter described at a broader view of gender-related behavior. Expectation states theory makes a similar argument, suggesting that sex differences disappear when explicit information is provided about participants' status (Berger \& Zelditch, 1998). For example, Wood and Karten (1986) noted that women acted friendlier than men when competence levels of participants were unspecified but that this difference disappeared when women were described as the most competent in a mixed-sex group. Specific to our purpose, we predicted relatively small sex differences in smiling when females and males occupied the same role, were engaged in the same task, or had the same status and that these various contextual constraints would override gender-based norms for smiling behavior. We devised a number of moderators to assess the degree to which male and female participants were constrained by situations they were in. These included measures of general situational constraint, presence of identifiable social roles, and engagement with particular tasks.

The first moderator is in this group was overall situational constraint, which refers to the degree to which participants were in a structured or scripted social situation. The prediction was that the sex difference in smiling would be smaller when the immediate situation was an identifiable context with expectations about what 
was likely to transpire there. Moderators of situation constraint included several social roles and tasks. Three "occupational" roles were coded as moderators: teaching, caretaking, and interviewing. These roles are often used in studies of social behavior. Our prediction was that because these roles are associated with knowledge about what is typically expected and done in them, women and men would show more similar expressive behavior. In short, expectations about role-appropriate behavior were expected to prevail over gender norms for smiling leading to smaller sex differences in smiling.

We also considered a fourth social role, namely, power. A recurrent idea in the nonverbal communication literature is that those with lower power smile more as a display of deference or appeasement (Henley, 1977; Keating, 1985). However, empirical support for this idea has been found wanting. Although some studies appear to have found that smiling is associated with lower power (e.g., Deutsch, 1990), others have found this to be true only for some conditions (Dovidio, Brown, Heltman, Ellyson, \& Keating, 1988) or not true at all (J. A. Hall \& Friedman, 1999; Hecht \& LaFrance, 1998). Instead, our formulation contends that having or not having power entails adopting the appropriate role and that people know what is expected of them when they are in those roles. This should apply particularly to those in low-power roles, although it may even be the case for people who occupy high social power positions.

This argument differs somewhat from both the subordination hypothesis articulated by Henley (1977) and from J. A. Hall and Halberstadt's (1986) rendering of the dominance-status hypothesis. Henley (1977) argued that sex differences in nonverbal behavior directly parallel differences in power such that women smile more than men because more smiling is required by low-status individuals, and women have lower status than men. J. A. Hall and Halberstadt tested whether the sex difference in smiling was different when participants had different levels of power. In contrast, we predicted that people in low social-power roles, regardless of sex, would show similar smiling behavior. When people are on more equal footing with each other, then they revert to the default position that calls for women and men to adopt different expressive behavior, specifically different amounts of smiling.

We proposed that situational constraints operated also when participants were required to carry out particular tasks. Moderators were devised to characterize four tasks used frequently in social interaction research. These included deception (participants instructed to lie), competition, conflict (participants instructed to take an oppositional stance), and persuasion. Similar to the predictions made for social roles, we expected that sex differences in smiling would be smaller when males and females were given the same task to perform.

\section{Emotion Salience}

The third factor conjectured to affect the size of sex differences in smiling concerns the degree to which participants were in a situation in which feelings, especially negative ones, were running high. In such contexts we expected to find larger sex differences in smiling because dealing with emotions is a domain thought to be more the province of women than men. Hochschild (1983) defined emotion work as "the management of feeling to create a publicly observable facial and bodily display" (p. 7), and she proposed that emotion work is gendered work. Such emotion work is gendered either because women are socialized to attend to the emotional needs of others or because they are steered to roles, pursuits, and occupations in which emotion work is a key element (Frith \& Kitzinger, 1998).

J. A. Hall and Halberstadt (1986) suggested in a related fashion that smiling under circumstances of high social tension may "be an attempt to be cheerful in spite of [the situation]" (p. 154) and that "women [may be] motivated to neutralize tense situations and to try to rescue all parties from the talons of social awkwardness" (p. 156). In short, smiling may be used to reverse or mitigate negative emotions. Consequently, we expected to find larger sex differences in smiling when emotion was a salient aspect of the social context. We devised several moderators to capture emotional salience: (a) social tension, (b) task tension, (c) self-disclosure, (d) embarrassment, (e) sadness, (f) happiness, and (g) humor.

We distinguished between two distinct types of tension: social tension and task tension. Social tension was designed to capture the kind of tension that was evident when there was interpersonal conflict or notable unease. In contrast, task tension involved situations in which participants anticipated being evaluated on the basis of their performance. We predicted a smaller sex difference in both situations, although it seemed likely that the sex difference in smiling would be larger when there was marked social tension because such situations are likely to call for more emotion work.

Situations involving self-disclosure were also conceptualized as precipitating fairly high levels of emotion and the accompanying need to regulate it, because revealing personal and intimate details can be an intense emotional experience (e.g., Keltner \& Bonnano, 1997). Consequently, we predicted larger sex differences in smiling in self-disclosing situations. Finally, we proposed a series of moderators designed to capture the type of emotion that was salient. Studies were coded for the presence of (a) embarrassment, (b) sadness, (c) happiness, and (d) humor. Anger, disgust, surprise, and pain were proposed as moderators but these states did not occur in more than one or two studies and were dropped from further analyses. We predicted larger sex differences in smiling in response to the negative emotions (embarrassment and sadness) than to the positive emotions (happiness and humor) because in the former, there is more need for emotion work.

In sum, our perception of sex differences in smiling was that the size of the difference would show considerable variability across people and contexts. In particular, we hypothesized that sex differences in smiling would be larger when gender norms were activated and when the immediate situation was characterized by heightened emotion. In contrast, we expected that men and women would be much more similar in how much they smiled when they were in the same role or required to carry out the same task.

\section{Method}

\section{Retrieval of Studies}

We searched the empirical literature for studies that documented a quantitative relationship between sex and smiling, even if that relationship was not the central one of the investigation. Along with published articles, unpublished materials such as conference papers, theses, dissertations, and other unpublished papers were included. This was done to counter the publication bias toward positive results (Rosenthal, 1979) and to obtain the most complete and reliable estimate possible. For unpublished reports, 
authors were contacted on the basis of their listed institutional affiliation. Dissertations were obtained from University Microfilms International.

We used several methods for obtaining relevant reports. First, computer searches using the key words smile, smiles, and smiling were conducted with the following databases: PsycINFO (1967-2001), Sociofile (19742001), MEDLINE (1975-2001), ERIC (1966-2001), Social Science Citation Index (1973-2001), Anthropological Index (1975-2001), and Dissertations Abstracts International (1861-2001). Several other abstract resources, not electronically available, were searched using these key words as well as nonverbal communication and facial expression. Second, several reference lists were searched for relevant studies. These included bibliographies of research on nonverbal behavior (Davis, 1972; Davis \& Skupien, 1982; Obudho, 1979; Patterson, Reidhead, Gooch, \& Stopka, 1984; Russell \& Fernandez-Dols, 1997; Thorne \& Henley, 1975), nonverbal communication texts (Argyle, 1988; Canary \& Dindia, 1998; Knapp \& Hall, 1997; LaFrance \& Mayo, 1978; Philippot, Feldman, \& Coats, 1999), several texts on gender (Eagly, 1987; Eakins \& Eakins, 1978; J. A. Hall, 1984; Matlin, 1993; Perry, Turner, \& Sterk, 1992), a review of nonverbal signs of deception (Zuckerman \& Driver, 1985), impressions created by nonverbal behavior (DePaulo, 1992; Tickle-Degnen, Hecht, Harrigan, Ambady, \& Rosenthal, 1997), gender and emotion (Fischer, 2000), and expectancy effects (J. A. Hall \& Briton, 1993; M. J. Harris \& Rosenthal, 1985).

Third, conference programs for the American Psychological Association (APA), American Psychological Society, Western Psychological Association, Eastern Psychological Association, Midwestern Psychological Association, Rocky Mountain Psychological Association, and Society for Personality and Social Psychology (up until 2000) were manually searched for relevant reports. Fourth, we included the smile studies used in J. A. Hall \& Halberstadt's (1986) meta-analysis and the studies of nonsocial smiling mentioned in J. A. Hall (1984) as well as in J. A. Hall's (1998) update. Fifth, we searched with the ancestry method, in which the reference lists of retrieved studies are scanned for additional reports (Rosenthal, 1991). Finally, we used the invisible college method (Rosenthal, 1991), whereby individual investigators known to have conducted research on facial expression (e.g., Buck, Ekman, Levenson) and nonverbal behavior (e.g., Feldman, Riggio) were contacted via e-mail with requests to furnish relevant data.

\section{Criteria for Selection}

Several selection criteria were then applied to the obtained studies. The first requirement was that there be a measure of smiling by both sexes. The measure of smiling could be smile frequency, smile duration, or ratings of facial pleasantness. Measures were averaged if more than one smiling estimate was reported. The rationale for averaging measures into a single index was that a nonsignificant difference in effect size was obtained when individual measures were compared. There was no difference in observed effect size when both frequency and duration measures were used ( $d \mathrm{~s}=0.76$ and 0.71 , respectively; $Q_{\mathrm{W}}=0.36$ ). There was also no difference in effect size when studies that used frequency measures were compared with those that used pleasantness ratings ( $d=0.40$ in each case).

We excluded studies in which participants were instructed to smile or to move their faces a certain way and ones that measured degree of facial expressiveness or animation because such expressions could include nonsmiling facial displays. Also excluded were studies that used actors or clinical populations (e.g., Brennan-Parks, Goddard, Wilson, \& Kinnear, 1991).

We also excluded studies of children under the age of 13 years. This was done for both substantive and practical reasons. First, there is no evidence of sex differences in smiling in young children (J. A. Hall \& Halberstadt, 1986). But more to the point, the primary aim in this analysis was to assess whether sex differences in smiling behavior are moderated by a host of situational moderators, such as teaching, caretaking, and interviewing, most of which are not applicable to children's lives. Our goal was also to focus on moderators around which there has been substantial inquiry and debate, for which there is no obvious parallel in children, such as interviewing someone. In short, many of our moderators cannot be assessed in studies of children's facial expression because the contexts for these studies and the methods used in them are not codable using the set of moderators proposed here. Finally, the size of empirical literature bearing on sex differences in smiling in adults is now quite large. Were this meta-analysis to include the very large number of studies that have measured smiling in infants and children, the resulting analysis would likely be unmanageable. In sum, although there is good reason to look for the point at which children begin to reliably show sex differences in smiling and to examine potential moderators associated with it, that analysis warrants its own meta-analysis. Therefore, we sought to keep our review focused and realistic in scope by beginning with children aged 13 .

We eliminated duplicate reports of the same data such as those that occur when published reports follow unpublished ones. If a report did not provide a separate test of sex differences in smiling or if there were insufficient data to calculate an effect size, authors were requested to provide the relevant statistics or raw data. Of the approximately 120 requests made, 90 responses were received.

\section{Coding of Moderator Variables}

\section{Report Attributes}

In addition to coding theoretically relevant moderators, several aspects of the reports themselves were coded. These variables included publication date and observation date. The latter is relevant in the case of archival materials in which the coded data (e.g., yearbook photographs) might have been taken at an earlier time. For example, an experiment in a journal article published in 1990 might have used as data newspaper photos from the 1960s, in which case the publication date and the observation date would be different. If a study did not specify the year in which the material was collected, we coded it to be the same as publication date. Reports were coded as to their publication outlet (journal article, chapter in an edited volume, monograph, conference paper, unpublished paper, thesis, or dissertation). Also coded were sex of first author, sex of experimenter, and possible feminist orientation of first author (coded for those authors who were listed as members in the Psychology of Women Division [Division 35] of the APA).

Finally, we noted whether studies differentiated between smile types, specifically the distinction between Duchenne smiles (smiles reliably associated with positive emotion) and nonDuchenne smiles (Ekman, 1985; Ekman, Davidson, \& Friesen, 1990; Ekman \& Friesen, 1982). Given the current framework, specifically the idea that smiling may often be less a sign of underlying emotion than a display adopted to achieve social goals, it seemed possible that the sex difference in smiling would be larger for non-Duchenne smiles than for Duchenne smiles. In other words, it seemed more likely that males and females would show comparable amounts of Duchenne smiling when they were truly feeling happy. This distinction turned out to be relatively rare in our database, with only eight reports reporting amounts of both Duchenne and non-Duchenne smiles. When we compared the sex difference for these two smile types, the effect sizes were not significantly different from each other, $Q_{\mathrm{B}}(1)=3.14$. Thus, we did not include this variable in subsequent analyses. 


\section{Gender Norms for Smiling}

Because no study of sex differences in smiling directly manipulated or measured gender norms for smiling, we coded eight moderators that served as proxies for these, namely, conditions that were theorized to activate concerns about behaving in a gender-appropriate way.

Four levels of observation awareness were coded. The highest level of observation awareness was coded if an operating video camera or observer was visible to participants. The next level of observation awareness was coded if participants were informed about the presence of observers or cameras but they could not see them. The third level of observation awareness was coded if there was ambiguity about whether participants were being recorded. This might occur, for example, if a consent form mentioned that their behavior would be recorded but there were no further indications of such, or if there was a one-way mirror but no reference was made to it. The least amount observation awareness was coded if participants were kept unaware as to whether they were being recorded or if participants were unobtrusively observed in the field.

Presence of others involved a simple count of the number of others with whom the participant actively interacted: no others, one other, two or three others, or four or more others. Situations in which the participant was overtly photographed were coded as one other present. The experimenter was not counted unless interaction with that person was part of the experimental manipulation.

Engagement with others assessed how much participants were interactionally involved with others. The following four types rather than levels of engagement were coded: unengaged (e.g., others may have been present but they were occupied with something else), coaction (e.g., parallel activity, as in watching a movie together), active interaction (e.g., debating, problem solving), and imagined other (e.g., delivering a speech to a camera).

Three types of instructions to get acquainted were coded. One type involved situations in which participants might have felt some inclination to introduce themselves to one another, such as being together in a waiting room, but there was no explicit stipulation that they do so. In a second type, participants were given explicit instructions to get acquainted with another person. The default category included situations in which getting acquainted was not relevant.

The research setting moderator categorized whether the observations were collected in the laboratory or in the field. Archival materials distinguished between facial expression materials collected from existing records such as advertisements or yearbook photos (archival) or from direct observation of people in naturalistic or laboratory settings (nonarchival). Archival posing was applicable to those studies that drew on existing visual records. A distinction was made between other-directed posing (e.g., advertisements) and self-posing (e.g., selecting pictures from an array). A candid shot category was included initially, but only a very few cases were found. Finally, familiarity between participants was coded as one of four levels: (a) no prior contact; (b) slightly acquainted, such as having had one or two brief prior encounters; (c) moderately acquainted, such as colleagues, coworkers, and social acquaintances; and (d) familiar, such as family, close friends, and roommates.

\section{Demographic Moderators}

Nationality. Participants from the United States made up the majority of studies. Five studies involved Canadians. Twenty-one effect sizes were grouped as Asian by the following countries of origin: Japan (6), China (3), Korea (8), and India (2). Eighty-two effect sizes described samples from Europe: Belgium (25), Germany (25), England (22), Italy (4), Spain (4), and Sweden (2). Five effect sizes came from Australian samples.

Ethnicity. We coded ethnicity for U.S. participants as Caucasian, African American, Native American, Asian, (Chinese, Japanese, and Korean and Indian) and Australian Aboriginal. Initially, there was a Hispanic code, but this category contained no entries. For both nationality and ethnicity, there was a default category for studies in which participants in a single study were members of more than one cultural group or where ethnicity information was unavailable.

Partner demographics. If no information was provided about the partner or if there was no primary partner (such as in yearbook photographs), partner characteristics were coded as unknown. Partner ethnicity was coded as Caucasian, African American, Asian, or mixed. Ethnicity composition was coded as same, different, or mixed. Partner sex was coded as male, female, both, or unknown. "Both" included studies in which participants had partners of both sexes or in which some participants interacted with one sex while others interacted with the other sex but the statistical results yielded only one average effect size across conditions. Sex composition coded whether an interaction involved those who were same-sex, opposite-sex, both, and unknown.

Participant age. We coded age as being in one of the following categories: 13-17 years, 18-23 years, 24-64 years, and 65 or more years. These age groups correspond to commonly used categories in the nonverbal communication literature. If the article did not report age but described the population in terms of level in school (e.g., high school students, college students), the average age corresponding to the school level was assigned (e.g., age 18-23 for college students). If the article noted that the sample consisted of adults, these were coded in the 24-64-year-old group, as were persons described as middle-aged, graduate students, or adults in public settings such as airports or conventions.

Partner age. The categories for partner age were 1-12 months, 13-59 months, 5-9 years, 10-12 years, 13-17 years, 18-23 years, 24-64 years, and unknown. There were no studies in which the partner age was 13-17 years or 13-59 months, so these categories were dropped from subsequent analyses. If there were several partners of varying ages, partner age was coded as mixed. The partner age relationship coded whether the partner was younger than the participant or the same age or older or unknown.

\section{Situational Constraints on Smiling}

Three groups of moderators were devised to capture different types of situational constraint. They included overall situational constraint, social roles, and assigned tasks. Overall situational constraint involved a binary judgment as to whether participants were in a structured or scripted social situation. For example, studies in which participants were asked to role play were coded as having overall situational constraint.

Four roles were coded: power, caretaking, teaching, and interviewing. We coded power in terms of the participant having one of 
three types: more power than the partner, equal power with the partner, or less power than the partner. ${ }^{2}$ Higher power was coded if the participant had the capacity to reward, hire, promote, inform, praise, punish, impose penalties, or withhold some benefit from another. Equal power was coded for participants that did not differ explicitly in terms of assigned power. These could be friends, peers, or coworkers. Low power was coded for those having lower resource control, such as by being an employee, subordinate, or student. Interviews were coded as equal power unless the interviewer had the clear capacity to reward or withhold from the interviewee as in a job interview. Persuasion attempts and photo sessions were coded as equal power unless there was clear indication of differential control or resources. Participants in teaching roles were coded as having high power. Thus, power and teaching were not mutually exclusive.

Three other social roles, namely caretaking, teaching, and interviewing were coded as being present or absent. Tasks too were coded as being present or not. Tasks included deception, conflict, competition, and persuasion. The activities of waiting for the experiment to begin or having a conversation were not coded as tasks because they were not seen as clearly involving consensual practices.

\section{Emotion Salience}

Several moderators were devised to characterize emotion-laden interactional contexts. The emotion salience moderators were tension (social and task), self-disclosure, and specific emotion states (sadness, embarrassment, happiness, and humor).

Two types of tension were coded that were regarded as orthogonal. Three levels of social tension were coded: (a) social tension (e.g., arguing), (b) no apparent level of tension or comfort, and (c) social comfort (e.g., relaxed interactions as in restaurants or parks). Similarly, three levels of task tension or evaluation apprehension were coded: (a) task tension (e.g., participants' interviewing skills were being evaluated), (b) no apparent task tension or comfort, and (c) comfort or relaxation with a task (e.g., playing a game). Self-disclosure was coded as present if participants were explicitly asked to disclose personal details or if there was a general emphasis on self-disclosure within the context of the interaction (e.g., people interacting with a therapist). Studies requiring people to get acquainted were not coded as self-disclosing. Finally, we also coded studies by the presence of the following emotion states: (a) embarrassment, (b) sadness, (c) happiness, and (d) humor. Anger, disgust, surprise, and pain were initially part of the coding system, but these affective states did not occur in more than one or two studies and hence were dropped from further analyses.

\section{Coder Reliability}

Two waves of data collection took place. The first wave included studies dated prior to and including 1994, and the second wave included studies from 1995 to mid-2001. Two groups of raters did the coding, one group for each wave of data collection. Each group of coders consisted of four people with two raters of each sex coding each moderator. None of the authors of this report coded any of the studies in the first wave of the coding and Elizabeth Levy Paluck was one of the four coders for the second wave of the coding. Coders used photocopied excerpts of the
Table 1

Coding Reliability for the First and Second Waves of Studies

\begin{tabular}{lrrr}
\hline & \multicolumn{2}{c}{ Sample } & \\
\cline { 2 - 3 } \multicolumn{1}{c}{ Variable coded } & \multicolumn{1}{c}{ Mean $r$} \\
\hline Sex of experimenter & .79 & 1.00 & .89 \\
Camera visibility & .96 & .94 & .95 \\
Presence of others & .75 & .95 & .85 \\
Engagement & .91 & .87 & .89 \\
Getting acquainted & .91 & .84 & .87 \\
Research setting & .86 & .99 & .93 \\
Acquaintanceship & .98 & .98 & .98 \\
Age & .81 & .95 & .88 \\
Ethnicity & .93 & 1.00 & .97 \\
Partner age & .98 & .95 & .97 \\
Partner age relationship & .84 & .92 & .88 \\
Partner ethnicity & .86 & .71 & .77 \\
Ethnicity composition & .84 & .83 & .83 \\
Partner sex & .96 & .94 & .95 \\
Sex composition & .94 & .94 & .94 \\
Deception & .96 & 1.00 & .98 \\
Competition & .83 & .89 & .86 \\
Conflict & .46 & .96 & .71 \\
Teaching & .85 & 1.00 & .93 \\
Interview & .93 & .92 & .92 \\
Persuasion & .87 & .93 & .90 \\
General situational constraint & .83 & .83 & .83 \\
Archival posing & .96 & .84 & .90 \\
Power & .88 & .85 & .86 \\
Self-disclosure & .88 & .91 & .89 \\
Embarrassment & .91 & .89 & .90 \\
Social tension & .86 & .85 & .85 \\
Task tension & .74 & .68 & .71 \\
Humor & .94 & .98 & .96 \\
Other assigned emotions (collapsed $r$ ) & .79 & .92 & .86 \\
\hline
\end{tabular}

Note. Variables not shown were coded by Marvin A. Hecht only because they required information only available outside of the Method section.

Method section from each study, which concealed the identity of the authors, the theoretical orientation of the study, and the results. If a report described a study containing more than one experimental condition, each condition was coded separately.

Table 1 provides separate reliability measures for the first sample, the second sample, and the combined average of the two samples. Reliability ranged from .71 to 1.00 . Reliability for both waves of data collection was handled in two stages. First, each coder coded each study independently. Interrater reliability was calculated by taking the aggregate reliability of the four coders at each time using the Spearman-Brown formula (Rosenthal \& Rosnow, 1991). This formula are designed for continuous ratings. For categorical variables, we calculated reliability by breaking codes into a series of $0 / 1$ columns ( $R$. Rosenthal, personal communication, May 15, 1994). On the basis of these results, we eliminated several variables that had low agreement (e.g., "tone of situation" was dropped).

\footnotetext{
${ }^{2}$ Several dimensions were initially constructed to tap situations in which people were assigned to roles differing in social power. These were power status, vulnerability, and need to please. However, because we subsequently found these ratings to be highly correlated with one another, the single power variable was used.
} 
Overall reliability was remarkably high. For example, across all codes in the second sample, the four coders were unanimous in their assigned codes $85.5 \%$ of the time. For another $9.9 \%$ of the codes, three of the four coders were in agreement, and in these cases the deviant code was changed to reflect the majority. This left $4.6 \%$ of the total codes in which coders were split down the middle. Thus, phase two of the coding procedure entailed a group discussion in which coders discussed classifications with the goal of strict adherence to the original coding system. If consensus could not be reached, Marianne LaFrance served as tiebreaker.

\section{Computation of Effect Sizes}

The effect size index reported is Cohen's $d$, defined as the difference between the means for female and male participants divided by the pooled within-sex standard deviation. Positive values for $d$ signify greater smiling by females than by males. Cohen's $d$ is an excellent measure to use when reporting sex difference research because it is readily interpretable, referring to differences between the sexes in standard deviation units. An effect size of 0.20 indicates a two-tenths of a standard deviation unit difference between males and females (Cohen, 1977).

When researchers reported only $t \mathrm{~s}$ and degrees of freedom, or reported only the product-moment correlation values $(r s)$, we calculated the effect size using formulas recommended by Rosenthal (1991, pp. 17-20, equations 2.14, 2.21). In cases in which the sex difference was calculated using a dyad as the unit of analysis (e.g., studies using married couples), we used the formula for correlated observations (Rosenthal, 1991, p. 15, equation 2.13) to calculate the effect size because a within-subject analysis of variance is typically used for dyadic analyses (see Kenny \& la Voie, 1985). If researchers provided the total sample number $(N)$ but not the specific numbers of males and females $(n \mathrm{~s})$, we used N/2. Rosenthal (1991, pp. 17-18) has shown that when the female to male ratio is no more than $70: 30$, this formula leads to an estimate within $8 \%$ of the effect size calculated with the "true" $n$ s.

We calculated the overall $d$ with and without the unknown effect sizes assigned a value of zero. Because the primary goal of this meta-analysis was to investigate whether the sex difference in smiling depended on moderator variables that sometimes varied across conditions within a single study, we followed the established practice of preserving relevant between-conditions effect sizes (see Eagly, Chen, Chaiken, \& Shaw-Barnes, 1999; Eagly, Karau, \& Makhijani, 1995; B. T. Johnson \& Eagly, 2000). Of the total number of reports described here, less than one fourth contributed more than one effect size to the moderator analysis. In short, the typical effect size represents males and females compared in a single condition or situation. Nevertheless, appropriate caution should be used interpreting these analyses, because they challenge the assumption of effect size independence (cf. B. T. Johnson \& Eagly, 2000, p. 518). Because the calculation of withinstudy effect sizes sometimes leads to $d$ s with relatively small $n \mathrm{~s}$, we corrected for small sample size using a formula recommended by Hedges (1981): $d=J(m) g$, where $g$ is the uncorrected effect size and $J(m) \approx 1-(3 /(4 \times d f)-1)$. If multiple measures of smiling were used, ${ }^{3}$ we combined the measures using the procedure developed by Rosenthal and Rubin (1986). When the correlation between measures was not specified by the authors, we converted the effect sizes to Fisher's $Z$, averaged the measures, and
Table 2

Summary Statistics for the Meta-Analysis

\begin{tabular}{ll}
\hline \multicolumn{1}{c}{ Statistic } & \multicolumn{1}{c}{ Value } \\
\hline Total no. of participants & 109,654 \\
Research reports & 162 \\
No. of effect sizes $(k)$ & $418^{\mathrm{a}}$ \\
Overall mean weighted effect size $(d)$ & 0.41 \\
95\% confidence interval & $0.39,0.42$ \\
Overall mean weighted $d$ (including assigned zeros) & 0.40 \\
Unweighted mean $d$ & 0.38 \\
Unweighted median $d$ & 0.41 \\
Sum of $Z$ & 948.86 \\
Combined $Z$ & 46.41 \\
Probability associated with mean $Z$ & $1.7 \times 10^{-470}$ \\
Fail-safe $N$ from file drawer analysis & 332,267 \\
\hline
\end{tabular}

${ }^{\mathrm{a}} k=448$ effect sizes with the inclusion of assigned zeros.

then converted back to the effect size, as suggested by Rosenthal (1991).

After the mean weighted effect size was tested for significance (as indicated by a $95 \%$ fixed effects confidence interval not including zero), the homogeneity of the effect sizes was tested by $Q_{\mathrm{W}}$, which has an approximate chi-square distribution with $k-1$ degrees of freedom, where $k$ is the number of effect sizes. A significant $Q_{\mathrm{W}}$ means that the set of effect sizes tested is heterogeneous. Heterogeneity indicates that the variability of the effect sizes is not due to sampling error alone (Hedges, 1994).

If the summary analysis of the effect sizes indicated heterogeneity, we conducted tests for the moderator variables. This analysis was achieved by dividing the effect sizes into categories and then comparing their mean effect sizes. This comparison was done by computing $Q_{\mathrm{B}}$, which has an approximate chi-square distribution with $p-1$ degrees of freedom, where $p$ is the number of categories within each moderator variables (Hedges, 1994; Hedges \& Olkin, 1980). We then computed contrasts to determine which categories differed significantly from one another. The critical value for the chi-square was 3.84 at $p<.05$ with 1 degree of freedom.

\section{Results}

\section{Characteristics of Sample}

A total of 109,654 participants were included in 162 reports yielding 448 effect sizes-418, excluding assigned-zero effect sizes. All subsequent analyses are based on the latter number, namely 418. Analysis yielded a mean weighted effect size of 0.41 with a $95 \%$ confidence interval of 0.39 to 0.42 indicating that females smiled more than males. The median effect size was also 0.41 , with the unweighted mean effect size a little lower ( $d=0.38$ ), but both were within the range of the $95 \%$ confidence interval for the mean weighted effect size (see Table 2). The mean weighted effect size, which included assigned zeros, yielded a

\footnotetext{
${ }^{3}$ Measures were typically frequency and duration; however, most studies used frequency alone, and duration was always supplied in addition to frequency, usually by coders scoring smiling using the facial action coding system (Ekman \& Friesen, 1978). This made a comparison between studies measuring frequency versus studies measuring duration impossible.
} 
similar value of 0.40 , with a $95 \%$ confidence interval of 0.39 to 0.41 .

Table 3 shows the stem and leaf plot of effect sizes. Note that the effect sizes are relatively normally distributed. Note too that the effect sizes ranged from -2.05 , indicating substantially greater smiling by males, to 2.42 , indicating substantially greater smiling by females. A vote count of the stem and leaf results revealed 69 effect sizes for which males smiled more than females and 4 effect sizes that had a true zero value. The majority, namely 345 effect sizes, showed that females smiled more than males.

The variation in effect sizes was confirmed by a homogeneity analysis which indicated that the set of effect sizes was heterogeneous, $Q_{\mathrm{W}}(417)=2,072.01, p<.0001$. Hence, examination of potential moderator variables is warranted.

We also calculated the combined probability of the effect sizes. The combined $Z$ was 46.41 , and the $p$ value associated with this

Table 3

Stem and Leaf Diagram of Effect Sizes

\begin{tabular}{|c|c|}
\hline Stem & Leaf \\
\hline 2.5 & \\
\hline 2.4 & 2 \\
\hline 2.3 & 4 \\
\hline 2.2 & \\
\hline 2.1 & 9 \\
\hline 2.0 & \\
\hline 1.9 & 5 \\
\hline 1.8 & 39 \\
\hline 1.7 & \\
\hline 1.6 & 24 \\
\hline 1.5 & 26 \\
\hline 1.4 & 3 \\
\hline 1.3 & 244459 \\
\hline 1.2 & 00134668 \\
\hline 1.1 & 12235678 \\
\hline 1.0 & 13345668889999 \\
\hline 0.9 & 02233333578999 \\
\hline 0.8 & 0022444556677899 \\
\hline 0.7 & 11112334445567778999 \\
\hline 0.6 & 0112222233334444566666666677778899 \\
\hline 0.5 & 0001112222333333344445555666677788899 \\
\hline 0.4 & 000011112222222233333444455555566666777777778899 \\
\hline 0.3 & 001111111222223333444444444555555556666778889999 \\
\hline 0.2 & 0011112222333333444444455556666777778889999999 \\
\hline 0.1 & 00111355666789999 \\
\hline 0.0 & 00000000000000000000000000000000000222233444567788899 \\
\hline-0.0 & 12234555668 \\
\hline-0.1 & 001199 \\
\hline-0.2 & 0223566789 \\
\hline-0.3 & 11289 \\
\hline-0.4 & 2349 \\
\hline-0.5 & 024577 \\
\hline-0.6 & 3 \\
\hline-0.6 & 01158 \\
\hline-0.7 & 346 \\
\hline-0.8 & 158 \\
\hline-0.9 & 028 \\
\hline-1.0 & 06 \\
\hline-1.1 & 1 \\
\hline-1.2 & 8 \\
\hline-1.3 & 225 \\
\hline-1.4 & \\
\hline-1.5 & 7 \\
\hline-1.6 & \\
\hline-1.7 & 5 \\
\hline-1.8 & 4 \\
\hline-1.9 & 5 \\
\hline-2.0 & 5 \\
\hline-2.1 & \\
\hline-2.2 & \\
\hline-2.3 & \\
\hline-2.4 & \\
\hline-2.5 & \\
\hline
\end{tabular}


was highly statistically significant. Next, we conducted a file drawer analysis to determine how many unobtainable studies there would have to be for the combined $p$ to be no longer significant (Rosenthal, 1979, 1991). This number is known as the fail-safe $N$. In the present meta-analysis, there would have to have been 332,267 unavailable studies, which seems unlikely.

\section{Impact of Moderator Variables}

\section{Report Attributes}

Table 4 displays the results for several report attributes. Because a few of these variables contained more than two levels or categories, contrasts were computed between the mean weighted effect sizes to allow interpretation of significant between-classes effects (Hedges \& Olkin, 1980).

Publication date. About a third of the studies were conducted between 1965 and 1980, a second third were conducted in the early 1980s, and the last third were conducted from 1985 to mid-2001. The analysis of publication date produced a significant betweenclasses effect, $Q_{\mathrm{B}}(5)=7.47, p<.01$; however, there was no significant linear trend for publication date. The size of the sex difference in smiling varied by decade of publication, with the smallest occurring for reports published during the $1970 \mathrm{~s}$ $(d=0.37)$ and the largest occurring in the decade preceding that, the 1960 s $(d=0.50)$, but this contrast was not significantly different $\left(Q_{\mathrm{W}}=1.09\right)$.

Observation date. Because some reports involved visual records taken prior to the date they were published, we next examined whether there was a significant change in effect size that was due to the dates of the materials themselves. This analysis produced a significant between-classes effect, $Q_{\mathrm{B}}(5)=11.83, p<$ .05 . However, once again, there was no evidence of a linear trend $\left(Q_{\mathrm{W}}=0.32\right)$. Also, like the findings for publication date, the largest effect size occurred for the 1960s $(d=0.46)$, which was larger than for the 1970s $(d=0.43)$, but again, this was not statistically significant $\left(Q_{\mathrm{W}}=0.71\right)$. The effect size for the $1970 \mathrm{~s}$ was significantly larger than for the $1980 \mathrm{~s}\left(d=0.38 ; Q_{\mathrm{W}}=7.10\right.$, $p<.05)$; it was also larger than that for the 1990s $(d=0.41)$, but there is no clear pattern of a systematic increase or decrease over time in the sex difference in smiling.

Publication outlet. Journal articles constituted the major source of effect sizes, but nearly a third came from other sources in the following order: books, chapters, unpublished conference papers, and other unpublished papers. Analyses showed a significant between-classes effect, $Q_{\mathrm{B}}(5)=41.35, p<.001$. Conference papers produced the largest effect size $(d=0.68)$ and other

Table 4

Tests of Moderators of Effect Sizes (ds) for Report Attributes

\begin{tabular}{|c|c|c|c|c|c|c|}
\hline Variable & $Q_{\mathrm{B}}$ & $k$ & $n$ & Mean weighted $d$ & $95 \% \mathrm{CI}$ & $Q_{\mathrm{W}}$ \\
\hline Publication date & 7.47 & & & & & \\
\hline $1960 \mathrm{~s}$ & & 4 & 262 & 0.50 & $0.25,0.75$ & 1.50 \\
\hline 1970s & & 87 & 9,249 & 0.37 & $0.32,0.41$ & $297.10 * * * *$ \\
\hline $1980 \mathrm{~s}$ & & 199 & 33,869 & 0.43 & $0.40,0.45$ & $762.99 * * * *$ \\
\hline $1990 \mathrm{~s}$ & & 128 & 68,326 & 0.40 & $0.38,0.41$ & $1,002.94 * * * *$ \\
\hline Observation date & $11.83 * *$ & & & & & \\
\hline $1930 \mathrm{~s}$ & & 6 & 1,144 & 0.45 & $0.33,0.57$ & $52.46 * * * *$ \\
\hline $1950 \mathrm{~s}$ & & 9 & 1,265 & 0.37 & $0.25,0.48$ & 11.97 \\
\hline $1960 \mathrm{~s}$ & & 19 & 4,435 & 0.46 & $0.40,0.52$ & $67.71 * * * *$ \\
\hline 1970s & & 94 & 18,905 & 0.43 & $0.40,0.46$ & $456.45^{* * * *}$ \\
\hline $1980 \mathrm{~s}$ & & 165 & 28,186 & 0.38 & $0.35,0.40$ & $721.10 * * * *$ \\
\hline 1990s & & 125 & 55,719 & 0.41 & $0.39,0.43$ & $750.50 * * * *$ \\
\hline Publication outlet & $41.35 * * * *$ & & & & & \\
\hline Journal & & 292 & 98,108 & 0.40 & $0.38,0.41$ & $1,653.96 * * * *$ \\
\hline Chapter & & 9 & 502 & 0.39 & $0.21,0.57$ & 6.19 \\
\hline Book & & 25 & 4,014 & 0.47 & $0.41,0.54$ & $55.54 * * * *$ \\
\hline Conference & & 13 & 2,606 & 0.68 & $0.60,0.76$ & $34.28 * * * *$ \\
\hline Unpublished & & 32 & 1,384 & 0.21 & $0.10,0.32$ & $58.31 * * *$ \\
\hline Theses & & 47 & 3,040 & 0.40 & $0.32,0.47$ & $222.39 * * * *$ \\
\hline Sex of first author & $48.11 * * * *$ & & & & & \\
\hline Female & & 229 & 76,926 & 0.36 & $0.33,0.38$ & $982.70 * * * *$ \\
\hline Male & & 176 & 31,088 & 0.43 & $0.42,0.44$ & $1,021.76 * * * *$ \\
\hline Unknown & & 13 & 1,640 & 0.19 & $0.09,0.29$ & 19.44 \\
\hline Sex of experimenter & $18.81 * * * *$ & & & & & \\
\hline Female & & 15 & 909 & 0.38 & $0.25,0.52$ & $30.70 * * *$ \\
\hline Male & & 22 & 1,478 & 0.41 & $0.30,0.51$ & $54.68 * * * *$ \\
\hline Unknown & & 377 & 107,276 & 0.40 & $0.39,0.52$ & $1,966.67 * * * *$ \\
\hline $\begin{array}{c}\text { Feminist orientation } \\
\text { of first author }\end{array}$ & 2.49 & & & & & \\
\hline Member of Div. 35 & & 42 & 9,488 & 0.37 & $0.33,0.41$ & $188.13^{* * * *}$ \\
\hline Nonmember & & 376 & 100,166 & 0.41 & $0.40,0.80$ & $1,951.40 * * * *$ \\
\hline
\end{tabular}

Note. $Q_{\mathrm{B}}=$ between-classes effect; $k=$ number of effect sizes; $\mathrm{CI}=$ confidence interval; $Q_{\mathrm{W}}=$ homogeneity within each class; Div. 35 = Division 35 of the American Psychological Association, the Psychology of Women Division.

$* * p<.05$. *** $p<.01$. ***** $p<.001$. 
Table 5

Intercorrelations Between Theoretically Meaningful Moderators

\begin{tabular}{|c|c|c|c|c|c|c|c|c|c|c|}
\hline Variable & 1 & 2 & 3 & 4 & 5 & 6 & 7 & 8 & 9 & 10 \\
\hline 1. Observation awareness & - & & & & & & & & & \\
\hline 2. Presence of others & .01 & - & & & & & & & & \\
\hline 3. Engagement with others & $-.26 * * * *$ & $.34 * * * *$ & - & & & & & & & \\
\hline 4. Instructions to get acquainted & -.08 & .01 & .06 & - & & & & & & \\
\hline 5. Research setting & .04 & $.21 * * * *$ & $.15 * * *$ & $-.18 * * * *$ & - & & & & & \\
\hline 6. Archival material & $-.38 * * * *$ & -.05 & $.16^{* * * *}$ & $-.16^{* * * *}$ & $.47 * * * *$ & - & & & & \\
\hline 7. Familiarity & $-.20 * * * *$ & $.29 * * * *$ & .02 & $-.14 * * *$ & $.37 * * * *$ & -.07 & - & & & \\
\hline 8. Overall constraint & $-.18 * * * *$ & $-.13 * * * *$ & $.13 * * *$ & -.05 & $-.28 * * * *$ & $-.14 * * *$ & $-.28 * * * *$ & - & & \\
\hline 9. Power & $-.25 * * * *$ & -.06 & $.14 * * *$ & $-.12 * *$ & .06 & $-.12 * * *$ & $.23 * * * *$ & $.35 * * * *$ & - & \\
\hline 10. Caretaking role & -.04 & .02 & .09 & -.08 & $.11^{* *}$ & $-.22 * * * *$ & $.52 * * * *$ & $-.10 * *$ & $.33 * * * *$ & - \\
\hline 11. Teaching & -.01 & .01 & .03 & -.03 & -.05 & -.08 & -.04 & .01 & $.20 * * * *$ & $.29 * * * *$ \\
\hline 12. Interview & -.07 & .04 & $.12 * * *$ & -.04 & $-.36 * * * *$ & $-.30 * * * *$ & $-.28 * * * *$ & $.33 * * * *$ & $.26 * * * *$ & -.08 \\
\hline 13. Deception & -.01 & -.06 & .03 & -.04 & $-.12 * * *$ & $-.10 * *$ & -.08 & $.19 * * * *$ & -.03 & -.05 \\
\hline 14. Competition & -.06 & $.34 * * * *$ & -.04 & -.02 & $.13 * * *$ & -.06 & $.27 * * * *$ & -.08 & -.01 & .05 \\
\hline 15. Conflict & -.03 & .01 & .03 & -.03 & -.09 & -.08 & $.30 * * * *$ & .03 & -.07 & $.16^{* * * * *}$ \\
\hline 16. Persuasion & -.05 & .01 & .04 & -.05 & $-.15^{* * *}$ & -.09 & -.10 & $.23 * * * *$ & $.15 * * *$ & -.06 \\
\hline 17. Social tension & .07 & -.04 & -.03 & $-.31 * * * *$ & $.31 * * * *$ & $.12 * * *$ & $-.53 * * * *$ & $-.35 * * * *$ & .03 & .23 \\
\hline 18. Task tension & .05 & -.08 & -.03 & -.08 & $.23 * * * *$ & $.20 * * * *$ & $.15 * * *$ & -.29 & -.08 & $.12 * *$ \\
\hline 19. Self-disclosure & -.01 & .02 & .08 & -.05 & $-.18 * * * *$ & -.22 & .02 & $.11 * *$ & .04 & $.27 * * * *$ \\
\hline 20. Embarrassment & .01 & -.02 & -.05 & -.04 & $-.14 * * *$ & $-.12 * *$ & -.10 & $.14 * *$ & $-.11 * *$ & -.06 \\
\hline 21. Sadness & -.05 & $.22 * * * *$ & .02 & -.04 & $-.13^{* *}$ & $-.10 * *$ & -.05 & $.15 * * *$ & .06 & .06 \\
\hline 22. Happiness & -.03 & $-.14 * * *$ & $-.20 * * * *$ & -.05 & -.04 & .00 & $.19 * * * *$ & .00 & -.04 & -.00 \\
\hline 23. Humor & $.10^{* *}$ & -.10 & $-.52 * * * *$ & -.04 & $-.13 * * *$ & $-.11 * *$ & -.05 & -.01 & $-.10 * *$ & -.06 \\
\hline
\end{tabular}

$* * p<.05 . \quad * * * p<.01 . \quad * * * * p<.001$.

unpublished, that is, non-peer-reviewed, papers, the smallest ( $d=$ 0.21 ). It is perhaps noteworthy that there was a statistically significant contrast when unpublished reports were compared with all other outlets $\left(Q_{\mathrm{W}}=19.25, p<.05\right)$, with the latter group producing larger effect sizes. However, when journal articles were compared with all other outlets, the contrast was not significant $\left(Q_{\mathrm{w}}=1.69\right)$.

Sex of first author. There was clear evidence that sex of first author moderated the sex difference in smiling, $Q_{\mathrm{B}}(2)=48.11$, $p<.001$. When men were the first author, the effect size $(d=0.43)$ was significantly larger than when women were the first author $\left(d=0.36 ; Q_{\mathrm{W}}=29.02, p<.05\right)$. This result has also been observed for studies of sex differences in other social domains, although the reasons for it are unknown (e.g., Eagly \& Carli, 1981; Wood, 1987).

Sex of experimenter. In the majority of studies, it was not possible to definitively determine the sex of the person or persons actually conducting the studies from reading the Method section. Fewer than $9 \%$ of the reports provided information as to experimenter sex. For the data that were available, the results mirror that for author sex; the sex difference in smiling was greater for studies that had male experimenters $(d=0.41)$ than those in which the experimenter was a woman $(d=0.38)$, although now the effect was not statistically significant $\left(Q_{\mathrm{W}}=0.08\right)$.

Possible feminist orientation of first author. There was no evidence that feminist orientation of the first author, measured as being a registered member of Division 35 of the APA, moderated the size of the sex difference in smiling, $Q_{\mathrm{B}}(1)=2.49$. Note, however, that coding on this moderator could only be applied to first authors who were members of Division 35 of the APA.

\section{Tests of Theoretically Relevant Moderators}

Table 5 shows the bivariate correlations of the moderators with one another.
Correlations among moderators. We devised a number of independent moderators to capture situational features that were predicted to affect how much males and females smile. Table 5 displays all the theoretically relevant bivariate correlations among the moderators.

Consider the moderators addressing gender norms for smiling (e.g., observational awareness, engagement with others). Even though each individual moderator was devised to capture a factor that might activate more concern about displaying gender-appropriate smiling behavior (and hence increase the size of the sex difference in smiling), we created the complete set of these gender-norm moderators to capture different circumstances through which this might happen. In fact, we found few correlations between moderators within this group. For example, the correlation between observation awareness and presence of others was .01, and the correlation between familiarity and engagement with others was .02.

Within the set of situational moderators, on the other hand, we expected some degree of association between the single measure of overall situational constraint and some roles and tasks because roles and tasks by their very nature constrain behavior. For example, we found that overall situational constraint was modestly correlated with the caretaking role $(r=-.10)$ and with the deception task $(r=.19)$.

For the emotion salience factor, we separately measured social tension and task tension on the grounds that they reflect different kinds of emotion salience. More specifically, we conjectured that task tension would be higher when participants had to achieve or complete some assigned activity but would not be much in evidence in unstructured encounters. Although social tension and task tension were significantly associated with each other $(r=.24)$, the pattern of correlations with other features of the context indicates 


\begin{tabular}{|c|c|c|c|c|c|c|c|c|c|c|c|c|}
\hline 11 & 12 & 13 & 14 & 15 & 16 & 17 & 18 & 19 & 20 & 21 & 22 & 23 \\
\hline-.01 & -.07 & -.01 & -.06 & -.03 & -.05 & .07 & .05 & -.01 & - & -.05 & -.03 & .10 \\
\hline .01 & .04 & -.06 & $.34 * * * *$ & .00 & .01 & -.04 & -.08 & .02 & -.02 & $-.22 * * * *$ & $-.14 * * *$ & $-.10 * * *$ \\
\hline .03 & $.12 * * *$ & .03 & -.04 & .03 & .04 & -.03 & -.02 & .08 & -.05 & .02 & $-.20 * * * *$ & $-.52 * * * *$ \\
\hline-.03 & -.04 & -.04 & -.02 & -.03 & -.05 & $-.31 * * * *$ & -.08 & -.05 & -.04 & -.04 & -.05 & -.04 \\
\hline-.05 & $-.36 * * * *$ & $-.12 * * *$ & $-.12 * * *$ & -.09 & $-.14 * * *$ & $.31 * * * *$ & $.23 * * * *$ & $-.18 * * * *$ & $-.14 * * *$ & $-.11 * * *$ & .04 & $-.13 * * *$ \\
\hline-.08 & $-.30 * * * *$ & $-.10 * *$ & -.06 & -.08 & -.09 & $.12 * *$ & $.20 * * * *$ & $-.22 * * * *$ & $-.12 * * *$ & $-.10 * *$ & .00 & $-.11 * *$ \\
\hline-.04 & $-.28 * * * *$ & -.08 & $.27 * * * *$ & $.30 * * * *$ & -.10 & $.53 * * * *$ & $.15^{* * *}$ & .02 & -.10 & -.05 & $.19 * * * *$ & -.05 \\
\hline $.12 * *$ & $.33 * * * *$ & $.19 * * * *$ & -.08 & .03 & $.23 * * * *$ & $-.35^{* * * * *}$ & $-.29 * * * *$ & $.11^{* *}$ & $.14 * * *$ & $.15^{* * *}$ & .00 & -.01 \\
\hline $20 * * * *$ & $.26 * * * *$ & -.03 & -.01 & -.07 & $.16^{* * * *}$ & .03 & -.08 & .04 & $-.11 * *$ & .06 & -.04 & $-.10 * *$ \\
\hline $.29 * * * *$ & -.08 & -.05 & .05 & $.16 * * * *$ & -.06 & $.28 * * * *$ & $.12 * * *$ & $.27 * * * *$ & -.06 & .06 & -.01 & -.06 \\
\hline - & & & & & & & & & & -.02 & -.03 & -.02 \\
\hline-.06 & - & & & & & & & & & -.03 & -.11 & .07 \\
\hline-.02 & .08 & - & & & & & & & & -.02 & -.04 & -.03 \\
\hline-.01 & -.05 & -.02 & - & & & & & & & -.02 & $.09 * *$ & -.02 \\
\hline-.02 & -.06 & -.02 & -.01 & - & & & & & & -.02 & -.03 & -.02 \\
\hline-.02 & $.17 * * * *$ & .06 & -.02 & -.02 & - & & & & & -.03 & -.04 & -.03 \\
\hline .03 & $-.37 * * * *$ & .01 & -.07 & -.08 & $-.15 * * *$ & - & & & & -.09 & .01 & -.05 \\
\hline$-.10 * *$ & $-.26 * * * *$ & $-.31 * * * *$ & $-.14 * * *$ & -.05 & $-.27 * * * *$ & $.24 * * * *$ & - & & & -.02 & .06 & .06 \\
\hline-.04 & $.28 * * * *$ & -.06 & -.04 & $.15 * * *$ & -.07 & $-.32 * * * *$ & .03 & - & & $.10^{* *}$ & -.01 & $.13^{*}$ \\
\hline-.02 & $.22 * * * *$ & -.03 & -.02 & -.02 & -.03 & $-.19 * * * *$ & .04 & $.33 * * * *$ & - & -.03 & -.04 & $.32 * * * *$ \\
\hline-.02 & -.03 & -.02 & -.02 & -.02 & -.03 & -.09 & -.02 & $.10^{* *}$ & -.03 & - & & \\
\hline-.03 & $-.11 * *$ & -.04 & $-.09 * *$ & .03 & -.04 & .01 & .06 & -.01 & -.04 & -.03 & - & \\
\hline-.02 & .07 & -.03 & -.02 & -.02 & -.03 & -.05 & .06 & $.13 * * *$ & $.32 * * * *$ & -.02 & .10 & - \\
\hline
\end{tabular}

that they were associated with different features of the situation. For example, task tension was significantly correlated with engaging in a competitive task $(r=-.14)$, as expected, but social tension was not $(r=-.07)$.

In our framework, we conceived gender norms, situational constraints, and emotion salience as being independent contributors to the size of the sex difference in smiling. For instance, we theorized that observation awareness would be associated with a larger sex difference in smiling by making gender norms for this expressive behavior more salient. As such, we did not expect that observation awareness would be associated with emotional salience. In fact, if observational awareness were found to be significantly correlated with social tension, then an argument could be made that observation awareness moderated the sex difference effect size not because of its association with gender norms (as we argue) but rather because of its association with self-consciousness and possible resulting anxiety or tension. However, we found observation awareness to be uncorrelated with social tension $(r=.07)$. Thus, it appears that increased scrutiny activates greater adherence to normative behavior more than it makes participants socially tense.

In contrast, we expected and found a significant negative relationship between observational awareness and familiarity $(r=$ -.20 ), because both increase scrutiny and the presence of strangers was theorized to increase concern about behaving in a genderappropriate way. Nonetheless, the modest association between them indicates grounds for assessing their individual impacts on the overall effect size.

Regression analysis. We next entered all theoretically relevant moderators into a regression analysis. Specifically, we conducted a simultaneous weighted multiple regression to determine the association of each moderator with the overall $d$ while controlling for all other moderators (Knight, Fabes, \& Higgins, 1996). If a moderator included an unknown or not applicable code, it was recoded so as to remove these nonmeaningful levels from the analysis. Categorical variables with more than two levels were recoded into dichotomous variables to represent the presence or absence of the theoretically relevant factor. For example, the moderator "presence of others" contained five levels, each representing how many people participants were interacting with. This moderator was recoded as "other person(s) present" (1) versus "no one else present" $(0)$. The overall effect size was highly significant $\left(R^{2}=.19, p<.0001\right)$. Standardized regression coefficients for the moderators were, on average, small in size. Nonetheless, with four exceptions (instructions to get acquainted, competition, sadness, and humor), all the moderators were significantly related to the overall effect size. Thus, although some moderators were intercorrelated, the overall pattern was one in which many factors independently affected the size of the sex difference in smiling (see Table 6).

In the following sections, results are presented for the three primary moderator clusters: gender norms, situational constraints on smiling, and emotion salience.

\section{Gender Norms for Smiling}

Table 7 presents the results for the moderators presumed to activate gender norms for smiling. Each moderator was created to capture the various ways the environment signals to participants that they might want to think about behaving in a genderappropriate way because each leads participants to believe their behavior is being viewed, monitored, or evaluated.

Observation awareness. As predicted, observation awareness produced a significant between-classes effect, indicating that as knowledge of being observed became more apparent, the effect 
Table 6

Standardized Regression Coefficients for the Association Between Each Moderator and the Smiling Difference (d)

\begin{tabular}{lc}
\multicolumn{1}{c}{ Moderator } & $\beta$ \\
\hline Observation awareness & $0.163 * * * *$ \\
Presence of others & $0.258 * * *$ \\
Engagement with others & $-0.159 * * *$ \\
Instructions to get acquainted & -0.006 \\
Research setting & $-0.038^{*}$ \\
Archival material & $-0.160^{* * * *}$ \\
Familiarity & $0.146 * * *$ \\
Overall constraint & $-0.055^{*} * * *$ \\
Power & $-0.132 * * * *$ \\
Caretaking role & $0.099 * * * *$ \\
Teaching & $-0.028 * * *$ \\
Interview & $0.134 * * *$ \\
Deception & $-0.078^{*} * * *$ \\
Competition & 0.002 \\
Conflict & $-0.118 * * * *$ \\
Persuasion & $-0.060^{*} * * *$ \\
Social tension & $-0.036 * *$ \\
Task tension & $-0.055^{*} * * *$ \\
Self-disclosure & $0.165 * * *$ \\
Embarrassment & $-0.039 * * * *$ \\
Sadness & 0.015 \\
Happiness & $-0.061^{*} * * *$ \\
Humor & 0.019 \\
\hline
\end{tabular}

$* p=.06 . \quad * * p<.05 . \quad * * * p<.01 . \quad * * * * p<.001$.

size favoring greater smiling by females became larger, $Q_{\mathrm{B}}(4)=$ 285.96, $p<.001$. The largest effect size was for the situations in which it was clear that the participants were being observed (e.g., overt video camera, $d=0.46$ ), and the smallest occurred when participants had no awareness of being observed $(d=0.19)$. The contrast comparing high awareness with all other situations in which observation was less obvious yielded a significant effect $\left(Q_{\mathrm{W}}=49.14, p<.05\right)$. So too was the contrast between situations in which participants were completely unaware of being observed with all other levels of awareness $\left(Q_{\mathrm{W}}=72.45, p<.05\right)$.

Presence of others. Concerns about presenting oneself as gender appropriate also led to the initial conjecture that the effect size would be greater when participants were in the company of more others rather than few or no others. This thinking was based on the idea that when one is alone there is relatively little need to use display rules to present a proper image to others (Buck, Losow, Murphy, \& Costanzo, 1992). The results, however, were contrary to this prediction. Although effect sizes varied as a function of how many others were present, $Q_{\mathrm{B}}(4)=71.77, p<.001$, the largest effect size occurred in the alone condition $(d=0.50)$. The smallest was evident for those contexts in which participants interacted with four or more others $(d=0.11)$. In short, sex differences in smiling decreased as the number of others increased. Some ideas about why this might have happened are considered below in our discussion of the results for the engagement with others moderator.

Engagement with others. The prediction for engagement with others was that the effect size for smiling would be largest when people were most involved in an interaction and smallest when they were not involved in a joint or parallel activity. Results clearly supported this hypothesis, $Q_{\mathrm{B}}(3)=61.93, p<.001$. When par- ticipants were not directly engaged with others, the effect size was very small $(d=0.08)$, showing that female and male participants smiled in near identical amounts. In contrast, the size of the sex difference in smiling for the coaction situation was larger ( $d=0.24)$, and for the active interaction situation, the effect size was larger still $(d=0.40)$. The contrast between the most engaged situation (face-to-face interaction) with the coaction situation (parallel activity) was statistically significant $\left(Q_{\mathrm{W}}=8.88, p<.05\right)$.

Yet, the largest effect size for the engagement with others moderator involved situations in which participants interacted with an imagined other, such as speaking directly to a camera $(d=0.63)$. These imagined-other situations, in which there was no real give-and-take with another person, produced a significantly larger effect size than when participants coacted with or actually engaged in a face-to-face interaction $\left(Q_{\mathrm{W}}=36.85, p<.05\right)$. At first pass, this finding appears somewhat surprising, as the largest effect size occurred when participants were alone. But this may help to explain it. It became clearer on closer inspection that of those conditions that were coded as alone (for the presence of others moderator), a substantial proportion included ones in which participants were asked to present themselves to an imagined other. In fact, the correlation between the presence of others alone code and the engagement with others imagined-other code was statistically significant $(r=.41, p<.0001)$. Therefore, a more accurate way of describing the alone code in the presence of others moderator was that participants were solitary and actively engaged, albeit with a noncorporeal other. Thus, it seems that alone does not mean removed from concerns about behaving in a genderappropriate way-quite the contrary. This code received the highest effect size because it activated cognizance of potential evaluation by one or more unseen others. As such, it is not surprising that these contexts precipitate feelings that one is being watched, which according to the gender-norms framework, would be associated with larger sex difference effect sizes.

Instructions to get acquainted. We predicted that explicit instructions to get acquainted would activate the motivation to behave in a gender-appropriate way, provoking larger sex differences in smiling. Although the sex difference effect size in smiling was greater $(d=0.53)$ when there were no explicit instructions to get acquainted $\left(d=0.37 ; Q_{\mathrm{W}}=4.05, p<.05\right)$, the overall between-classes effect was nonsignificant.

Research setting. Because laboratory contexts are likely to generate a greater sense that one is being monitored, our prediction was that the lab contexts would be associated with a larger effect size than field contexts. Results were generally supportive. There was a marginally significant between-classes effect, $Q_{\mathrm{B}}(1)=3.64$, $p<.06$, indicating a larger sex-based smiling difference in the lab $(d=0.43)$ than in the field $(d=0.40)$.

Archival data. Approximately three quarters of the effect sizes were based on observations taken on actual behavior, whereas the remaining quarter came from analysis of archival materials such as advertisements and yearbook photos. Drawing on Goffman (1979), who argued that print advertisements present idealized and polarized versions of gender roles, we considered it likely that archival materials would be associated with a higher sex difference effect size in smiling. We found support for this hypothesis: Studies that used archival materials reported a significantly larger effect size $(d=0.46)$ than nonarchival studies $(d=0.27)$, and this difference was significant, $Q_{\mathrm{B}}(1)=210.39, p<.001$. In addition, the posing 
Table 7

Tests of Moderators of Effect Sizes (ds) for Gender Norms of Expressiveness

\begin{tabular}{|c|c|c|c|c|c|c|}
\hline Variable & $Q_{\mathrm{B}}$ & $k$ & $n$ & Mean weighted $d$ & $95 \% \mathrm{CI}$ & $Q_{\mathrm{W}}$ \\
\hline Observation awareness & $285.96 * * * *$ & & & & & \\
\hline Clear awareness & & 295 & 84,560 & 0.46 & $0.45,0.47$ & $1,407.82 * * * *$ \\
\hline Knowledge of observation & & 49 & 2,176 & 0.40 & $0.31,0.48$ & $123.67 * * * *$ \\
\hline Possibility of observation/concealed observation & & 43 & 2,769 & 0.34 & $0.26,0.42$ & $129.73 * * * *$ \\
\hline Nonawareness & & 31 & 20,149 & 0.19 & $0.16,0.22$ & $127.93 * * * *$ \\
\hline Presence of others & $71.77 * * * *$ & & & & & \\
\hline 0 & & 37 & 11,090 & 0.50 & $0.46,0.54$ & $163.84 * * * *$ \\
\hline 1 & & 292 & 35,749 & 0.45 & $0.42,0.47$ & $943.40 * * * *$ \\
\hline $2-3$ & & 16 & 4,523 & 0.35 & $0.29,0.41$ & $53.11 * * * *$ \\
\hline 4 or more & & 7 & 272 & 0.11 & $-0.13,0.35$ & $12.63 * *$ \\
\hline Unknown or mixed & & 66 & 58,020 & 0.37 & $0.35,0.38$ & $832.60 * * * *$ \\
\hline Engagement with others & $61.93 * * * *$ & & & & & \\
\hline No engagement & & 12 & 607 & 0.08 & $-0.08,0.23$ & $32.27 * * *$ \\
\hline Coaction & & 13 & 1,400 & 0.24 & $0.13,0.35$ & $36.67 * * * *$ \\
\hline Interaction & & 360 & 104,525 & 0.40 & $0.42,0.39$ & $1,822.01 * * * *$ \\
\hline Imagined other & & 33 & 3,122 & 0.63 & $0.56,0.70$ & $119.13 * * * *$ \\
\hline Instructions to get acquainted & 4.45 & & & & & \\
\hline Not applicable & & 377 & 107,394 & 0.40 & $0.39,0.42$ & $1,976.73 * * * *$ \\
\hline First encounter but no instructions & & 18 & 1,200 & 0.37 & $0.25,0.48$ & $37.73 * * *$ \\
\hline Explicit instructions & & 23 & 1,060 & 0.53 & $0.41,0.66$ & $53.11 * * * *$ \\
\hline Research setting & $3.64^{*}$ & & & & & \\
\hline Lab & & 263 & 22,916 & 0.43 & $0.40,0.83$ & $804.59 * * * *$ \\
\hline Field & & 154 & 88,638 & 0.40 & $0.39,0.41$ & $1,263.78 * * * *$ \\
\hline Archival material & $210.39 * * * *$ & & & & & \\
\hline Nonarchival & & 293 & 33,308 & 0.27 & $0.25,0.52$ & $918.07 * * * *$ \\
\hline Archival & & 125 & 76,364 & 0.46 & $0.45,0.48$ & $943.55 * * * *$ \\
\hline Posing in archival data & $207.80 * * * *$ & & & & & \\
\hline Posed by other & & 42 & 20,192 & 0.38 & $0.36,0.41$ & $370.46 * * * *$ \\
\hline Self-posed & & 81 & 55,806 & 0.49 & $0.48,0.51$ & $521.88 * * * *$ \\
\hline Nonarchival & & 295 & 33,656 & 0.28 & $0.25,0.30$ & $936.33 * * * *$ \\
\hline Familiarity & $53.46^{* * * * *}$ & & & & & \\
\hline No prior contact & & 248 & 59,310 & 0.45 & $0.43,0.46$ & $929.84 * * * *$ \\
\hline Slightly to moderately acquainted & & 23 & 2,417 & 0.42 & $0.33,0.50$ & $76.66^{* * * * *}$ \\
\hline Familiar & & 42 & 4,270 & 0.24 & $0.18,0.30$ & $110.25 * * * *$ \\
\hline Unknown & & 105 & 43,657 & 0.36 & $0.34,0.38$ & $901.79 * * * *$ \\
\hline
\end{tabular}

Note. $Q_{\mathrm{B}}=$ between-classes effect; $k=$ number of effect sizes; $\mathrm{CI}=$ confidence interval; $Q_{\mathrm{W}}=$ homogeneity within each class.

$* p=.06 . \quad * * p<.05 . \quad * * * p<.01 . \quad * * * * p<.001$

moderator indicated a significantly lower effect size when participants were constrained by another directing them to pose $(d=0.38)$ than when participants were free to select their own pose $(d=0.49), Q_{\mathrm{B}}(1)=207.80, p<.001$. When people are free to select their own pose, it appears that they draw on what they believe to be appropriate expressive behavior, and that appears to be that men should smile less than women.

Familiarity. We predicted that gender norms for expressiveness would be more salient during encounters with unfamiliar than with familiar others on the grounds that one is less concerned about societal norms in the company of friends than of strangers (Wagner \& Smith, 1991). Results bear this out. There was a significant overall between-classes effect for familiarity, $Q_{\mathrm{B}}(4)=53.46, p<.001$. The highest effect size $(d=0.45)$ occurred for those with no prior contact between participants, and the lowest ( $d=0.24$ ) was for those who were highly familiar with each other. This contrast was significant $\left(Q_{\mathrm{W}}=40.29, p<.05\right)$. The contrast comparing the most familiar others $(d=0.24)$ with somewhat familiar others was also significant $\left(Q_{\mathrm{W}}=11.20, p<\right.$ $.05)$. In short, it appears that familiarity reduces the inclination to behave in a gender-normative manner.

\section{Culture and Age Differences}

Table 8 displays results pertaining to whether culture and developmental stage of participants significantly moderated sex differences in smiling.

Nationality. Every country showed females to smile more than males; nonetheless, there was wide variation among nationalities in how large this difference was, $Q_{\mathrm{B}}(7)=246.28, p<.001$. The largest effect size occurred for Canadian participants, and the smallest for British nationals. The sex difference for both Asian and Australian participants evidenced an effect size between these two $(d=0.30)$. Studies using participants from the United States represented the largest proportion of all studies, and they showed an effect size of 0.45. A contrast of U.S. participants with all other nationalities was statistically significant $\left(Q_{\mathrm{W}}=7.08, p<.05\right)$. Ethnicity within the U.S. sample also proved to be a significant moderator, $Q_{\mathrm{B}}(6)=29.03, p<.001$. The largest sex difference in smiling appeared with Caucasians $(d=0.43)$, while smaller effect sizes were observed for African American $(d=0.25)$ samples. The contrast pitting Caucasians against all other ethnic groups was statistically significant $\left(Q_{\mathrm{W}}=7.07, p<.05\right)$. 
Table 8

Tests of Moderators of Effect Sizes (ds) for Culture and Age Characteristics of Sample

\begin{tabular}{|c|c|c|c|c|c|c|}
\hline Variable & $Q_{\mathrm{B}}$ & $k$ & $n$ & Mean weighted $d$ & $95 \% \mathrm{CI}$ & $Q_{\mathrm{W}}$ \\
\hline Nationality & $246.28 * * * *$ & & & & & \\
\hline American & & 293 & 78,242 & 0.45 & $0.44,0.47$ & $1,338.02 * * * *$ \\
\hline Canadian & & 5 & 232 & 0.59 & $0.33,0.86$ & 4.17 \\
\hline Asian & & 17 & 1,266 & 0.30 & $0.18,0.41$ & 17.16 \\
\hline Continental European & & 60 & 17,748 & 0.35 & $0.32,0.38$ & $384.43 * * * *$ \\
\hline England & & 22 & 391 & 0.13 & $-0.07,0.34$ & $57.81 * * * *$ \\
\hline Australian & & 5 & 482 & 0.30 & $0.12,0.48$ & 5.13 \\
\hline Far East (and India) & & 2 & 182 & 0.37 & $0.08,0.66$ & 0.06 \\
\hline Mixed & & 10 & 10,190 & 0.15 & $0.11,0.19$ & $17.78^{* *}$ \\
\hline Ethnicity & $29.03 * * * *$ & & & & & \\
\hline Caucasian & & 347 & 84,220 & 0.43 & $0.41,0.44$ & $1,685.59 * * * *$ \\
\hline African American & & 7 & 1,073 & 0.25 & $0.13,0.37$ & 5.02 \\
\hline Native American & & 1 & 150 & 0.27 & $-0.05,0.59$ & 0.00 \\
\hline Indian & & 2 & 1,266 & 0.37 & $0.08,0.66$ & 0.06 \\
\hline Asian & & 17 & 120 & 0.30 & $0.18,0.41$ & 17.16 \\
\hline Australian Aboriginal & & 1 & 182 & 0.22 & $-0.14,0.58$ & 0.00 \\
\hline Mixed & & 43 & 22,643 & 0.34 & $0.31,0.37$ & $335.14 * * * *$ \\
\hline Partner ethnicity & 5.89 & & & & & \\
\hline Caucasian & & 367 & 86,090 & 0.42 & $0.41,0.43$ & $1,748.68 * * * *$ \\
\hline African American & & 2 & 128 & 0.50 & $0.15,0.86$ & 0.57 \\
\hline Asian & & 19 & 1,448 & 0.31 & $0.20,0.41$ & 17.43 \\
\hline Mixed & & 30 & 21,988 & 0.35 & $0.32,0.37$ & $299.44 * * * *$ \\
\hline Ethnicity composition & $203.78 * * * *$ & & & & & \\
\hline Same & & 401 & 98,378 & 0.44 & $0.42,0.45$ & $1,828.32 * * * *$ \\
\hline Mixed & & 17 & 11,276 & 0.15 & $0.11,0.19$ & $39.91 * * * *$ \\
\hline Age & $248.08 * * * *$ & & & & & \\
\hline $13-17$ & & 41 & 16,446 & 0.56 & $0.53,0.59$ & $252.83 * * * *$ \\
\hline $18-23$ & & 176 & 42,445 & 0.45 & $0.43,0.47$ & $619.87 * * * *$ \\
\hline $24-64$ & & 112 & 22,564 & 0.30 & $0.27,0.33$ & $340.26 * * * * *$ \\
\hline $65+$ & & 2 & 2,184 & 0.11 & $0.02,0.19$ & $8.53 * * *$ \\
\hline Unknown & & 87 & 26,015 & 0.35 & $0.33,0.37$ & $602.45 * * * *$ \\
\hline Partner age & $16.07 * * *$ & & & & & \\
\hline $1-12$ months & & 22 & 4,308 & 0.30 & $0.24,0.36$ & $49.04 * * * *$ \\
\hline 5-9 years & & 2 & 38 & 0.25 & $-0.40,0.89$ & 1.46 \\
\hline $10-12$ years & & 3 & 198 & 0.35 & $0.07,0.64$ & $6.85^{* *}$ \\
\hline $18-23$ years & & 121 & 9,189 & 0.40 & $0.36,0.44$ & $362.05 * * * *$ \\
\hline 24-64 years & & 61 & 3,692 & 0.32 & $0.25,0.39$ & $154.73 * * * *$ \\
\hline Unknown or mixed & & 208 & 92,169 & 0.41 & $0.40,0.43$ & $1,481.82 * * * *$ \\
\hline Partner age relationship & $-8.16^{* * * *}$ & & & & & \\
\hline Younger & & 28 & 1,966 & 0.34 & $0.26,0.43$ & 67.59 **** \\
\hline Same & & 99 & 8,307 & 0.44 & $0.39,0.48$ & $240.81 * * * *$ \\
\hline Older & & 26 & 980 & 0.37 & $0.24,0.50$ & $91.74 * * * *$ \\
\hline Unknown & & 265 & 98,401 & 0.40 & $0.39,0.42$ & $1,680.04 * * * *$ \\
\hline Partner sex & $176.49 * * * *$ & & & & & \\
\hline Female & & 26 & 5,476 & 0.29 & $0.23,0.34$ & $55.12 * * * *$ \\
\hline Male & & 29 & 1,582 & 0.55 & $0.45,0.65$ & $54.54 * * * *$ \\
\hline Both & & 206 & 25,328 & 0.27 & $0.25,0.30$ & $726.96 * * * *$ \\
\hline Unknown & & 157 & 77,268 & 0.45 & $0.44,0.47$ & $1,058.90 * * * *$ \\
\hline Partner sex composition & $155.45^{* * * *}$ & & & & & \\
\hline Same & & 45 & 1,781 & 0.48 & $0.39,0.58$ & $145.05 * * * *$ \\
\hline Opposite & & 53 & 5,154 & 0.35 & $0.30,0.41$ & $236.97 * * * *$ \\
\hline Both & & 166 & 24,908 & 0.27 & $0.25,0.30$ & $451.63 * * * *$ \\
\hline Unknown & & 154 & 77,811 & 0.45 & $0.44,0.46$ & $1,082.92 * * * *$ \\
\hline
\end{tabular}

Note. $Q_{\mathrm{B}}=$ between-classes effect; $k=$ number of effect sizes; CI $=$ confidence interval; $Q_{\mathrm{W}}=$ homogeneity within each class. $* * p<.05 . \quad * * * p<.01 . \quad * * * * p<.001$.

Age. Results show that participant age moderated the size of the sex difference in smiling, $Q_{\mathrm{B}}(3)=248.08, p<.0001$, resulting in a significant linear effect. The largest effect size was observed for teenagers $(d=0.56)$; this was smaller for young adults $(d=0.45)$, smaller still with middle-aged adults $(d=0.30)$, and smallest for adults older than $65(d=0.11)$. All contrasts between age groups were significant.
Partner age. Table 8 displays results showing that effect size was significantly affected by several partner attributes. First, partner age showed a significant between-classes effect, $Q_{\mathrm{B}}(5)=$ $16.07, p<.001$. Here, the relationship was not linear $\left(Q_{\mathrm{W}}=\right.$ $0.43)$; rather, the effect size was significantly larger when interaction partners were in the $18-23$ age group $(d=0.40)$ than when they were in the 24-64 age group $\left(d=0.32 ; Q_{\mathrm{W}}=4.29, p<.05\right)$. 
The partner age relationship also moderated the sex difference effect size $\left(Q_{\mathrm{B}}(3)=8.16, n s\right)$ and accounts for it. The effect size was larger when the participant and partner were the same age ( $d=0.44)$ than when the partner was younger $(d=0.34)$ or older $(d=0.37)$, although only the former contrast approached statistical significance $\left(Q_{\mathrm{W}}=3.61, p<.06\right)$. Because the preponderance of participants were in the 18-23-year-old group, it seems that peers are associated with larger effect sizes, whereas cross-age interactions are associated with lower effect sizes. In other words, women and men smile in more similar amounts when they are with people younger or older than themselves.

Partner sex was also a significant moderator, $Q_{\mathrm{B}}(3)=176.49$, $p<.001$. Specifically, the effect size was larger when the partner was male $(d=0.55)$ than when the partner was female $(d=0.29$; $\left.Q_{\mathrm{W}}=20.03, p<.05\right)$. In addition, the partner sex composition was a significant moderator, $Q_{\mathrm{B}}(3)=155.45, p<.001$. The effect size for same-sex pairs $(d=0.48)$ was significantly larger than for opposite-sex pairs $\left(d=0.35 ; Q_{\mathrm{W}}=5.32, p<.05\right)$. Thus it appears that same-sex pairings are associated with the largest tendency for the sexes to differ in how much they smile. Finally, neither partner ethnicity nor ethnicity composition of partner (same vs. other ethnicity) significantly influenced effect size $\left(Q_{\mathrm{W}} \mathrm{s}=3.90\right.$ and 1.64 , respectively).

\section{Situational Constraint}

Several lines of broad social psychological theorizing such as social role theory (Eagly, 1987) and expectation states theory (Berger \& Zelditch, 1998) propose that what looks like a sex difference may actually be a role or status difference. In the concrete realm of positive facial expression, we argue likewise, namely that men and women are more similar in how much they smile when they are in the same role or have the same status or are comparably constrained by the surrounding situation. Analyses of moderators testing this reasoning are shown in Table 9.

Overall constraint. Our prediction was that smiling differences between women and men would be smaller in situations in which behavior was prescribed or structured than in less constrained contexts. Results show this to be the case, $Q_{\mathrm{B}}(1)=3.82$, $p<.05$. The effect size was smaller $(d=0.38)$ when the situation was constrained in some fashion than when it was not $(d=0.41)$, although the absolute difference in the effect sizes is rather modest.

Roles. First we consider situations in which people had different levels of power or status vis-à-vis one another. As described earlier, J. A. Hall and Halberstadt (1986) reported that this moderator was not associated with sex differences in smiling. However, that analysis contained no studies in which participants

Table 9

Tests of Moderators of Effect Sizes (ds) for Situational Constraint

\begin{tabular}{|c|c|c|c|c|c|c|}
\hline Variable & $Q_{\mathrm{B}}$ & $k$ & $n$ & Mean weighted $d$ & $95 \% \mathrm{CI}$ & $Q_{\mathrm{W}}$ \\
\hline Overall constraint & $3.82 * *$ & & & & & \\
\hline Unconstrained & & 252 & 86,430 & 0.41 & $0.40,0.81$ & $1,379.82 * * * *$ \\
\hline Constrained & & 166 & 23,224 & 0.38 & $0.36,0.41$ & $688.38 * * * *$ \\
\hline \multicolumn{7}{|l|}{ Roles } \\
\hline Power & $3.90 * *$ & & & & & \\
\hline More & & 45 & 3,275 & 0.35 & $0.27,0.42$ & $91.78 * * * *$ \\
\hline Equal & & 304 & 93,217 & 0.42 & $0.40,0.43$ & $1,554.71 * * * *$ \\
\hline Less & & 69 & 15,214 & 0.35 & $0.32,0.39$ & $410.91 * * * *$ \\
\hline Caretaking & $14.89 * * * *$ & & & & & \\
\hline Absent & & 374 & 106,916 & 0.41 & $0.40,0.81$ & $1,941.15 * * * *$ \\
\hline Present & & 44 & 2,738 & 0.26 & $0.18,0.33$ & $115.97 * * * *$ \\
\hline Teaching & 0.61 & & & & & \\
\hline Absent & & 412 & 109,366 & 0.41 & $0.39,0.80$ & $2,062.18 * * * *$ \\
\hline Present & & 6 & 288 & 0.31 & $0.08,0.55$ & 9.22 \\
\hline Interview & $20.08 * * * *$ & & & & & \\
\hline No interview & & 334 & 106,119 & 0.40 & $0.39,0.41$ & $1,823.46 * * * *$ \\
\hline Interviews another & & 18 & 597 & 0.51 & $0.35,0.68$ & $30.15^{* *}$ \\
\hline Is interviewed & & 66 & 2,938 & 0.61 & $0.53,0.68$ & $188.33^{* * * * *}$ \\
\hline \multicolumn{7}{|l|}{ Tasks } \\
\hline Deception & 1.83 & & & & & \\
\hline Absent & & 408 & 110,974 & 0.41 & $0.39,0.80$ & $2,039.71 * * * *$ \\
\hline Present & & 10 & 732 & 0.28 & $0.11,0.46$ & $30.48^{* * * * *}$ \\
\hline Competition & 0.31 & & & & & \\
\hline Absent & & 414 & 109,580 & 0.41 & $0.39,0.80$ & $2,068.91 * * * *$ \\
\hline Present & & 4 & 74 & 0.27 & $-0.19,0.74$ & 2.80 \\
\hline Conflict & $11.41 * * * *$ & & & & & \\
\hline Absent & & 412 & 108,908 & 0.41 & $0.39,0.80$ & $2,055.15 * * * *$ \\
\hline Present & & 6 & 746 & 0.16 & $0.01,0.30$ & 5.46 \\
\hline Persuasion & $14.32 * * * *$ & & & & & \\
\hline No persuasion & & 404 & 108,196 & 0.40 & $0.39,0.79$ & $1,990.35 * * * *$ \\
\hline Required to persuade & & 14 & 1,458 & 0.61 & $0.50,0.72$ & $67.34 * * * *$ \\
\hline
\end{tabular}

Note. $Q_{\mathrm{B}}=$ between-classes effect; $k=$ number of effect sizes; $\mathrm{CI}=$ confidence interval; $Q_{\mathrm{W}}=$ homogeneity within each class.

*** $p<.05$. ***** $p<.001$. 
actually had less power than a partner, and consequently those authors were not able to adequately assess whether power moderated the relationship between sex and smiling. It is our contention that power does moderate the sex difference effect size by virtue of is capacity to constrain individuals' behaviors when they either have more or less power. Power or status is most often associated with recognized social roles (e.g., boss-employee, teacherstudent), and these roles prescribe expressive behavior, causing men and women to smile more similarly than when they are not in these roles. In particular, smiling differences should be smaller in conditions in which males and females have low power than when they have equal power. Such reasoning also suggests that the effect size for women and men who are in high-power roles should also be smaller than when they have equal power. In the latter case, high-power positions may afford more options than those in lowpower roles, but by virtue of being in an identifiable role, their behavior may still be more constrained than for people who are on more equal footing. The results shown in Table 9 support this idea, $Q_{\mathrm{B}}(2)=3.90, p<.05$. As predicted, the largest effect size in smiling occurred for participants with equal power $(d=0.42)$. Both the high-power context and the low-power context showed smaller effect sizes (both $d \mathrm{~s}=0.35$ ); that is, having or not having power reduced the difference in how much females smiled more than males. The difference between equal power and low power was significant $\left(Q_{\mathrm{W}}=12.21, p<.05\right)$, and the difference between equal power and high power approached significance $\left(Q_{\mathrm{W}}=3.13\right.$, $p=.06)$.

We also checked whether the effect sizes at different levels of power changed when only unacquainted participants were included. There is the possibility that the equal power category encompassed people who were well acquainted with one another while the high- and low-power categories encompassed those who knew each other much less well, resulting in the blurring of the contrast between power and familiarity. When only strangers were included in the analysis, the effect sizes for the equal- and lowpower conditions matched those when the whole sample was used ( $d \mathrm{~s}=0.45$ and 0.35 , respectively), although the statistical contrast was then no longer significant. It is worth noting that when only strangers were included, the effect size for high-power participants increased and was then much closer in absolute magnitude to the effect size for equal-power participants $(d=0.46$ vs. $d=0.45$, respectively).

For caretaking roles, we coded whether participants were in identifiable helpful roles such as doctors or therapists. Like other social roles, caretaking is constrained-people know what is expected. Consequently, we expected the sex difference in smiling would be smaller than when these roles were not in effect (Brody, 1997, 1999). Results supported this prediction - there was a significantly lower sex difference in smiling $(d=0.26)$ for those in caretaking roles than for those who were not $(d=0.41)$, $Q_{\mathrm{B}}(1)=14.89, p<.05$. The findings for teaching roles also followed the predicted pattern, with a smaller effect size for those in teaching roles $(d=0.31)$ than for those who were not $(d=0.41)$, but this contrast was not significant $\left(Q_{\mathrm{B}}=0.61\right)$. Interview roles did not significantly affect sex differences in smiling.

Tasks. Next we considered a range of tasks that participants were asked to do. We hypothesized that, like social roles, tasks often impose some ideas about how they should be performed, including the kind and degree of expressiveness that one should show while doing them. If so, then tasks should create smaller sex differences in smiling compared with not doing these prescribed activities. Here, the results were not entirely consistent with our argument. When participants were required to deceive or to compete, the effect sizes were in the right direction, that is, smaller, than when they were not required to take on these tasks (deception: $d=0.28$, no deception: $d=0.41$; competition: $d=0.27$, no competition: $d=0.41$ ), but the contrasts were not statistically significant. When participants were required to take an oppositional position, the prediction was confirmed (conflict: $d=0.16$, no conflict: $d=0.41), Q_{\mathrm{B}}(1)=11.41, p<.01$. When participants were required to persuade someone else, there was a significantly higher effect size $(d=0.61)$, which was contrary to the prediction $(d=0.40), Q_{\mathrm{B}}(1)=14.32, p<.05$. Persuasion actually was associated with a larger sex difference in smiling.

\section{Emotion Salience}

The third set of moderators centered on the prediction that the smiling difference between the sexes would be larger when emotion was salient on the grounds that emotional contexts tend to elicit emotion work and that women are more likely to do it. See Table 10 for these moderators.

Social and task tension. We predicted that when tension was present, females would smile more because it is often they who reduce it or who, at the least, want to reduce it (Leary \& Kowalski, 1995). In our coding system, we distinguished between social tension (nervousness about the social situation) and task tension (evaluation apprehension associated with task performance). As noted above, there was a modest correlation between them $(r=$ $.24)$.

There was an overall between-classes effect for social tension, $Q_{\mathrm{B}}(2)=204.87, p<.001$. Situations with high social tension were associated with a larger sex difference in smiling $(d=0.47)$ than were situations coded as comfortable $\left(d=0.20 ; Q_{\mathrm{W}}=\right.$ $191.99, p<.001)$ or coded as neutral $\left(d=0.43 ; Q_{\mathrm{W}}=10.68, p<\right.$ $.01)$. Task tension, on the other hand, was unrelated to the size of the sex difference ( $d=0.47$ for high task tension vs. $d=0.44$ for comfort with the task), $Q_{\mathrm{B}}(2)=-3.15, n s$. In other words, tension generated by the social context but not apprehension about task performance was associated with greater differences in smiling between the sexes.

Self-disclosure. Because self-disclosing is associated with high emotion (Dindia \& Allen, 1992), we predicted that it would be associated with women and adolescent girls smiling significantly more than men and adolescent boys. Self-disclosure did significantly moderate the sex difference effect size, $Q_{\mathrm{B}}(1)=$ $31.60, p<.001$. In self-disclosing contexts, the effect size was 0.64 compared with an effect size of 0.40 in non-selfdisclosing contexts.

Specific emotion states. We turn now to contexts in which specific emotion states were evident. We expected higher effect sizes when negative affect was present. We found that to be true for embarrassment - the difference was significantly greater when embarrassment was present $(d=0.70)$ than when it was not $(d=0.40), Q_{\mathrm{B}}(1)=11.63, p<.01$. Sadness, however, did not significantly affect the smiling difference effect size. Note that embarrassment but not sadness was correlated with social tension 
Table 10

Tests of Moderators of Effect Sizes (ds) for Emotion Salience Variables

\begin{tabular}{|c|c|c|c|c|c|c|}
\hline Variable & $Q_{\mathrm{B}}$ & $k$ & $n$ & Mean weighted $(d)$ & $95 \% \mathrm{CI}$ & $Q_{\mathrm{W}}$ \\
\hline Social tension & $204.87 * * * *$ & & & & & \\
\hline Tense & & 92 & 28,717 & 0.47 & $0.45,0.50$ & $434.70 * * * *$ \\
\hline Neutral & & 283 & 65,152 & 0.43 & $0.41,0.44$ & $1,230.03 * * * *$ \\
\hline Comfortable & & 43 & 15,785 & 0.20 & $0.17,0.23$ & $197.38 * * * *$ \\
\hline Task tension & -3.15 & & & & & \\
\hline Tense & & 78 & 4,990 & 0.47 & $0.41,0.53$ & $206.16^{* * * * *}$ \\
\hline Neutral & & 331 & 104,317 & 0.40 & $0.39,0.41$ & $1,842.84 * * * *$ \\
\hline Comfortable & & 9 & 347 & 0.44 & $0.22,0.65$ & $17.73 * * *$ \\
\hline Self-disclosure & $31.60 * * * *$ & & & & & \\
\hline Absent & & 371 & 107,158 & 0.40 & $0.39,0.79$ & $1,874.74 * * * *$ \\
\hline Present & & 47 & 2,496 & 0.64 & $0.56,0.72$ & $165.67 * * * *$ \\
\hline Embarrassment & $11.63 * * * *$ & & & & & \\
\hline Absent & & 405 & 109,064 & 0.40 & $0.39,0.80$ & $2,000.29 * * * *$ \\
\hline Present & & 13 & 590 & 0.70 & $0.53,0.87$ & $60.09 * * * *$ \\
\hline Sadness & 0.02 & & & & & \\
\hline Absent & & 409 & 109,307 & 0.41 & $0.39,0.80$ & $2,055.15^{* * * * *}$ \\
\hline Present & & 9 & 347 & 0.39 & $0.17,0.60$ & $16.84 * *$ \\
\hline Happiness & 0.28 & & & & & \\
\hline Absent & & 398 & 106,874 & 0.41 & $0.39,0.80$ & $2,012.87$ **** \\
\hline Present & & 20 & 2,780 & 0.39 & $0.31,0.46$ & $58.87 * * * *$ \\
\hline Humor & 1.94 & & & & & \\
\hline Absent & & 407 & 108,925 & 0.41 & $0.39,0.80$ & $1,988.17 * * * *$ \\
\hline Present & & 11 & 729 & 0.30 & $0.15,0.45$ & $81.90 * * * *$ \\
\hline
\end{tabular}

Note. $Q_{\mathrm{B}}=$ between-classes effect; $k=$ number of effect sizes; $\mathrm{CI}=$ confidence interval; $Q_{\mathrm{W}}=$ homogeneity within each class.

$* * p<.05 . \quad * * * p<.01 . \quad * * * * p<.001$.

( $r=.19$ vs. $r=.02$ ). We did not expect that positive emotion would moderate the size of the sex difference in smiling given that such situations do not call for emotion work, and that is what we found: Neither humor nor happiness significantly moderated sex differences in smiling.

\section{Discussion}

This meta-analysis confirms that women and adolescent girls reliably smile more than men and adolescent boys $(d=0.41)$, although it is worth noting that the sample included reports of instances in which the opposite was true. For example, there is some evidence that men smile more in response to feelings of disgust than do women (Ansfield, 1997).

According to Cohen (1977), effect sizes of 0.20, 0.50, and 0.80 indicate small, medium, and large effects, respectively. Thus, a $d$ of 0.41 constitutes a low to medium effect size and is lower than the $d$ of 0.63 reported in the initial meta-analysis of sex differences in smiling by J. A. Hall (1984). It is however closer to the effect size of 0.42 reported in a follow-up review with seven additional effect sizes (J. A. Hall \& Halberstadt, 1986). It is worth noting that an effect size of 0.41 is in the range of other reported sex differences. For example, sex differences in nonverbal sensitivity have been described as falling between 0.40 and 0.50 (J. A. Hall, 1998). J. A. Hall (1998) analyzed more than 300 effect sizes from several meta-analyses of sex differences and found that the modal effect size was a $d$ between 0.36 and 0.65. Canary and Hause (1993) summarized the results of 15 meta-analyses of sex differences and found that the effect size $(d)$ ranged from 0.03 to 0.40 . Thus, low to medium effect sizes for sex differences are common across various domains.

\section{Report Attributes}

Despite the consistency of the finding that men and adolescent boys smile less than women and adolescent girls, the size of this effect was significantly moderated by a host of variables. To begin with, several aspects of the reports themselves were associated with significant changes in effect size. For example, publication date was a significant moderator. Also in the case of archival materials, observation date significantly moderated the sex difference effect size. Nonetheless, there was no clear temporal trend for either moderator. The size of the sex difference has not systematically become smaller or larger with time. The historical variation underscores the need to explore the possibility that macrolevel factors (e.g., economic or political) may influence the degree to which the sexes differ in their smiling behavior.

The size of the smiling difference also varied as a function of where the report appeared and sex of the person who reported it. Conference papers reported the largest sex difference in smiling; unpublished papers reported the smallest sex difference, and published journal articles, which constituted the majority of the reports, had an effect size close to the complete sample. The sex of the first author also turned out to be a significant moderator. When the first author was a man, the sex difference showing male participants to smile less than female participants was larger than when the first author was a woman. Although the sex of the person conducting the data collection appeared not to affect the size of the sex difference, the number of studies that actually reported experimenter sex was exceedingly modest. There is no obvious explanation for why articles with men as first authors should be associated with larger sex differences in smiling. However, Eagly 
(1986) has suggested the intriguing possibility that differences associated with author sex may themselves be evidence for gender norms: "Gender difference research may be particularly vulnerable to some biases because researchers, as carriers of their society's culture, tend to have an elaborated set of beliefs about the nature of women and men" (p. 160).

\section{Theoretically Predicted Moderators of Differences in Smiling}

The moderator analysis clearly shows that the size of sex differences in smiling is largely contingent on group attributes and social conditions. Regarding group differences, the present results constitute a substantial reminder that gender is intricately interwoven with other social memberships (cf. Stearns, 1994). Even though women generally smiled more than men, the extent to which this was so varied by nationality, ethnicity, and age. Participants from the United States and Canada showed the largest sex differences in smiling compared with participants from the United Kingdom, for example. It is important to note here that this meta-analysis does not allow one to determine how much people in various countries actually smile; what it does reveal is how different the sexes are in the amount they do smile within particular cultures.

Nationality was a significant moderator, but the reasons why are unclear. One possibility is that sex differences in the display of positive emotion are expressed in different channels in different national groups. In other words, there may be cross-cultural consensus that women are the ones to express positive emotion, but in one culture that could take the form of greater smiling while in another it might be expressed through more vocal expressiveness. Another factor to consider is the degree to which different countries presume gender polarization. For example, it might be that sex differences in smiling are greater in countries that hold more strongly to the idea that the sexes are, or should be, very different in general, particularly with respect to expressiveness.

Ethnicity also mattered. Among African American participants, the effect size based on seven reports was significantly lower than that for Caucasian participants. Studies that examined both gender and ethnicity variations in expressive behavior in the same study were relatively few in number, yet there is some indication that sex differences in expressive behavior were more evident in some groups than in others. For example, Reid and Trotter (1993) found that among Caucasians, girls were more expressive toward a baby than boys, but there was no sex difference among the same-aged African American children.

Age too was found to be a significant moderator of the relationship between sex and smiling. The youngest group, namely adolescents (13-17 years), showed the largest effect size. It was smaller for young adults (18-23 years) and smaller still for older adults (24-65 years). This pattern is consistent with, although not identical to, the age-related results previously reported by J. A. Hall (1984). She reported a near zero effect size for infants and children and a significant effect size for adults. Our data set, comprising as it does more age distinctions, allows us to see that the relationship between age and smiling does not simply increase with age; rather, it appears to increase to a certain point after which it declines. This pattern of decreasing sex differences in smiling beyond adolescence is consistent with speculation from several sources that gender differentiation becomes increasingly important as individuals move from childhood toward early adulthood and declines thereafter (Birdwhistell, 1970; Brody, 1985; Saarni \& Weber, 1999).

These developmental results are compatible with both a socialization explanation and an evolutionary one. In terms of the former, children are seen to gradually acquire an understanding of display rules through socialization processes that direct girls to show positive emotion and boys to hide vulnerable ones (Cole, 1985). Moreover, sex differences in emotion (or its expressions) may serve the adaptive purpose of helping men and women fulfill different societal roles (Brody \& Hall, 1993). Socialization pressures may be particularly acute for adolescents and young adults as they move into sex differentiated societal roles but diminish thereafter.

Evolutionary theorists view facial expressions as adaptive (e.g., Fridlund, 1994; Izard, 1997). According to this perspective, sex differences evolved early in human history as the result of males and females facing different selective pressures based on their reproductive strategies. Because of their assumed greater parental investment, women presumably acquired the tendency to smile more than men as a means of establishing secure bonds with their mate and their children via communication of positive emotion (Alexander \& Wood, 2000). Although an evolutionary perspective might account for the phylogeny and ontogeny of sex differences in smiling, it does not, however, address why there might be cultural and situational variation in them.

Culture and situation also combined in the finding that the demographic attributes of partners moderated the sex difference effect size. For example, age of partner was a significant moderator such that sex differences in smiling were smaller when partners were babies, children, or older adults than when partners were adolescents and young adults. Given that many of the participants were in the latter two categories, what the results indicate is that children and mature adults elicit more comparable expressive behavior from males and females.

Sex of partner also moderated the sex difference in smiling. We found that same-sex dyads were associated with significantly larger effect sizes than opposite-sex pairs, a finding in line with previous research (J. A. Hall, 1984). We also found that male partners elicited a significantly larger sex difference in smiling than female partners. The combination suggests that gender norms for smiling are most in effect when people are with same-sex others and are modulated in the company of someone of the opposite sex. Recall again that this meta-analysis cannot determine how much people in various combinations actually smile; what it does indicate is how different the sexes are in how much they smile when they are in different gender combinations. Nonetheless, it appears that males smile the least when they are with other males. This could happen for any number of reasons. It may be that male-male interactions are more focused on dominance issues, and nonsmiling expressions tend to be rated as more dominant than smiling expressions (Keating, 1985). It may be that expressivity in general among men is less positively regarded (Fujita, Harper, \& Wiens, 1980). Darwin (1872/1965) noted that men often refrain from weeping in response to pain, perhaps because weeping is "thought weak and unmanly" (p. 153).

Gender norms. There is considerable support for the idea that the effect size favoring more smiling by women and adolescent 
girls and less smiling by men and adolescent boys is greater when people believe that someone is paying attention to them. The safest course in such circumstances would be to behave in genderappropriate ways (Deaux \& Major, 1987). The default option for women is to smile and for men not to. When participants were aware that their behavior was being observed, monitored, or evaluated, the sex difference effect size in smiling was significantly larger than when they believed they were unobserved. Specifically, when participants knew they were being observed, when they were in a laboratory context, when they were instructed to get acquainted, and when their behavior was for the record, women and adolescent girls smiled more than men and adolescent boys. Women are supposed to be communal and expressive (Brody \& Hall, 1993; Eagly, 1987; J. A. Hall, 1984), and smiling is an effective display for conveying these attributes. Men are supposed to be dominant and in control, and not smiling is one way to show that this is the case (Keating, 1985).

There were however some unexpected though not unintelligible findings. The largest effect size occurred for the alone code in the presence of others moderator and the imagined-other code for the engagement with others moderator. We initially thought that the sex difference in smiling would be minimized in the alone condition because then, we presumed, one would be less concerned about behaving in a gender-appropriate way. On closer inspection, such results appear to be quite compatible with the idea that there are gender display rules that call for women and adolescent girls to smile more than men and adolescent boys. First, the alone code was strongly correlated with the imagined-other code, supporting the idea that the alone condition was actually one in which participants were playing to an audience, albeit an unseen one. Thus, the alone condition appears to be like the "solitary" situations described by Fridlund and his colleagues. They found that people smile more in the imagined presence of someone they know than when they are not thinking about someone (Fridlund, 1991; Fridlund et al., 1990). In other words, interacting with imagined others may actually heighten concerns about behaving in a genderappropriate way, thus leading to the larger effect size.

Findings for the other contrast for the engagement with others moderator did support our initial thinking. When participants were actively engaged with others, the effect size was significantly larger than when they were in merely coaction (parallel) contexts, presumably because face-to-face interaction generates more gender-normative behavior. Finally, the very small effect size $(d=0.11)$ when participants were with four or more others might be understood as an indication that the presence of many others reduces the chance that one is likely to precipitate attention from others. In short, the presence of several others may be disinhibiting, reducing the pressure to behave in a gender-appropriate way. Familiarity may work similarly. When male and female participants were with others they knew well, the sex difference was quite small.

What ties these moderators together is the idea that participants adjust their behavior to be in compliance with gender-normative behavior. Gender display rules for smiling constitute the default option. When a situation is ambiguous with respect to appropriate behavior, then gender-role adherence is often the safest course; when a situation makes surveillance or assessment more salient, then gender-role adherence may be the obvious course. It thus appears that smiling is a small yet significant indication of sex-role identification and might actually be used as an unobtrusive indicator of such (LaFrance \& Carmen, 1980).

Situational constraints. There is also support for the idea that some contexts reduce sex differences in smiling by constraining everyone. Many situations have their own norms for appropriate expressive displays that apply to whomever is in that situation because a particular role needs to be filled or a particular constellation of behaviors need to be seen. For example, flight attendants are supposed to appear friendly and cheerful, whereas funeral directors are supposed to appear somber and reserved (Ashforth \& Humphrey, 1993). We found that sex differences in smiling were smaller when participants were coded as being constrained in some way. They were somewhat smaller when participants occupied some of the same roles. When participants were preoccupied with the same tasks however, there was little association with sex differences in smiling.

With regard to social roles, having low power and having high power were associated with smaller sex differences than when participants were not in a situation characterized by explicit differences in power. Because we did not want to arbitrarily exclude effect sizes, some of the equal-power effect sizes included interaction contexts in which power was not a relevant aspect of the overall environment and thus might have differed in ways besides power from the high- and low-power contexts. Nonetheless, other roles corroborated the pattern of smaller effect sizes occurring when people occupied particular roles.

Caretaking roles showed the same pattern. When males and females were in caretaking roles, the sex difference in smiling was significantly smaller than when they were not. This latter finding is particularly notable in light of the idea that some roles are clearly gendered and that one might therefore expect larger sex differences. For example, gender-in-context theorizing (Deaux \& Major, 1987) might lead one to expect that caretaking would be more strongly linked with women's roles and hence be associated with larger sex differences in expressive behavior. Such was not the case here. In fact, these results corroborate findings showing that when fathers in the United States are the primary caretakers, they are more nurturing and affectionate than fathers who are not (Pruett \& Lizenberger, 1992; Risman, 1987). Another related finding comes from observation of expressive display rules in different departments of an airline (Rutherford, 2001). "Cabin services," the branch of the airline industry that deals with human relations, was found to emphasize emotional expression within the department despite the fact that only a third of its managers were women. Moreover, members of this department were not likely to report there being sex differences in management style.

The reduced sex differences when men and women are assigned to the same social roles would be expected in light of both social role theory (Eagly, 1987) and expectation states theory (Berger \& Zelditch, 1998). According to social role theory, men and women by virtue of membership in the same social groups "experience common situational constraints because they tend to have the same or similar social positions within organizations or other structures such as families" (Eagly, 1987, p. 396). Similarly, expectation states theory holds that gender is a diffuse status characteristic such that women are assumed to have lower competence and status than men, other things being equal (Berger \& Zelditch, 1998). But when things are equal, then expectations for competence derive more from roles than sex. For example, Wood and Karten (1986) 
found that when provided with information only about the sex of other participants, members of experimentally formed groups believed that the men were more competent. Moreover, these beliefs translated into differential rates of instrumental and expressive behavior shown by men and women. Men showed more task behavior (e.g., more talking), and women showed more expressive behavior (e.g., more friendliness). In contrast, when specific and relevant status information not linked to gender was provided, the previously observed sex differences disappeared.

The present results, however, indicate that not every role significantly reduced sex differences in smiling. For example, teaching roles and interviewing roles reduced the sex difference but not significantly so. And it is certainly possible that there are roles that may be associated with large sex differences in smiling because one or the other sex is made especially uncomfortable. Yet the present data point to the power of social roles to produce behavioral uniformity.

When participants were required to engage in certain tasks, the results were decidedly more mixed. Being required to be oppositional did reduce the effect size, but the assignments to be deceptive and to compete reduced the effect size, although not significantly so. Moreover, the task of persuasion elicited the opposite effect such that the sex difference effect size was greater when the task involved being persuasive. As to the last, one possibility suggested by work on leadership indicates that women may first have to establish their gender credentials before trying to change someone's mind (i.e., demonstrate their ability to behave in gender-appropriate ways before exhibiting out-of-role behavior). For example, research shows that leadership is something that requires women to be more "social" than men (Eagly, Karau, \& Makhijani, 1995). In sum, some social roles appear to have the effect of overriding gender display rules whereas few tasks do. This distinction seems to be an important one. Many roles are well known even to those who have not had direct experience with them, and thus participants know what being in a role should look like. Tasks, on the other hand, tend to dictate what has to be done but not necessarily how it is to be done.

Emotion salience. The third factor believed to moderate how much the sexes smile relative to each other concerns whether emotion is a salient aspect of the prevailing environment. The idea is that women and adolescent girls smile more when the prevailing emotion climate is stressful. Results are generally supportive. For example, the sex difference in smiling favoring female participants was greater when the situation was characterized by social tension but not task tension. Effect sizes were also significantly larger when participants were involved in a self-disclosing situation and when the situation was marked by embarrassment. The presence of sadness did not moderate the smiling effect size nor did happiness or humor.

These findings partially corroborate the idea that the smiling sex difference becomes magnified when the situation highlights emotional stress. For example, recent work contends that women and adolescent girls do more "tending and befriending" in stressful situations (Taylor et al., 2000). Social tension also figured into J. A. Hall and Halberstadt's (1986) analysis of sex differences in smiling. In fact, in that meta-analysis, the social-tension hypothesis was the only one to receive strong support. Like them, we found that social tension significantly moderated sex differences in smiling. However, it appears that not every type of tension significantly moderates sex differences in smiling. Social tension moderated the sex difference effect size, but task tension did not. Moreover, observation awareness was not associated with social tension. In short, social tension seems to reflect a heightened emotional climate and not mere self-consciousness.

It might be argued that emotion-laden contexts are associated with the largest sex differences in smiling because women are simply more emotional. In other words, that social tension, selfdisclosure, and embarrassment were found to be significant moderators might be construed not as reflecting emotion work but as reflecting emotion nature-that women are more emotional. However, other studies have shown that men and women do not necessarily differ in their subjective reactions to emotional stimuli despite women's greater expressiveness (Kring \& Gordon, 1998; LaFrance \& Banaji, 1992; Wagner, Buck, \& Winterbotham, 1993).

\section{Conclusions}

The present meta-analysis had two general aims. First, we sought to assess whether sex differences in smiling would continue to be reliable in light of many more studies now available on the topic. Second, our aim was to consider a range of moderators not previously considered. With regard to the first, we found that women and adolescent girls do smile more than men and adolescent boys at a level commensurate with other studies of sex differences. With regard to the second, the regression analysis and the moderator analysis show that the extent of sex differences in smiling is highly contingent on social groups and social factors. Sex differences in smiling are culture and age specific. Activation of gender norms produces larger sex differences in smiling. Imposition of situational constraints sometimes reduces sex differences. And emotional climate, particularly evidence of heightened negative affectivity, is associated with larger differences between the sexes in how much they smile.

Throughout this article, we have alternately framed the results in terms of either females smiling more or males smiling less. This dual phrasing was done to acknowledge that sex differences in smiling could be described with equal accuracy either way because effect sizes describe difference and not behavioral magnitude. J. A. Hall (1987) correctly noted that describing group differences in terms of the group with the higher mean is an established convention. But she also noted that such convention might have the unfortunate consequence of neglecting factors that might specifically affect men's smiling. Finally, because the studies in the meta-analysis included noncommon features other than those variables being tested, we cannot draw the kind of causal conclusions that come from true experiments (Cooper, 1989). However, the results described here certainly point to factors that can then be experimentally tested in future research.

Herman Melville (1852/1949) suggested that the smile is the chosen vehicle for all ambiguities. What our meta-analysis has suggested instead is the smile is not so much ambiguous as it is a highly contingent social display. The sexes sometimes smile at dissimilar rates, and sometimes there appears to be a diminution of difference. To understand why requires looking beyond the differences between them.

\section{References}

References marked with an asterisk indicate studies included in the meta-analysis.

*Adams, R. M., \& Kirkevold, B. (1978). Looking, smiling, laughing, and moving in restaurants: Sex and age differences. Environmental Psychology and Nonverbal Behavior, 3, 117-121. 
Alexander, M. G., \& Wood, W. (2000). Women, men, and positive emotions: A social role interpretation. In A. H. Fischer (Ed.), Gender and emotion: Social psychological perspectives (pp. 189-210). Cambridge, England: Cambridge University Press.

*Amato, P. R. (1981). The effect of environmental complexity and pleasantness of prosocial behaviour: A field study. Australian Journal of Psychology, 33, 285-295.

*Ansfield, M. E. (1997). Paradoxical smiling during negative emotional experience: When a smile is a frown turned upside down. Unpublished manuscript, University of Virginia, Charlottesville.

Archer, J. (2000). Sex differences in aggression between heterosexual partners: A meta-analytic review. Psychological Bulletin, 126, 651-680. Argyle, M. (1988). Bodily communication (2nd ed.). London: Methuen.

Ashforth, B. E., \& Humphrey, R. H. (1993). Emotional labor in service roles: The influence of identity. Academy of Management Review, 18, $88-115$.

Banerjee, R., \& Lintern, V. (2000). Boys will be boys: The effect of social evaluation concerns on gender-typing. Social Development, 9, 397-408.

*Barnes, M. L., \& Rosenthal, R. (1985). Interpersonal effects of experimenter attractiveness, attire, and gender. Journal of Personality and Social Psychology, 48, 435-446.

*Bates, J. E. (1976). Effects of children's nonverbal behavior upon adults. Child Development, 47, 1079-1088.

Berger, J., \& Zelditch, M. (1998). Status, power and legitimacy: Strategies and theories. New Brunswick, NJ: Transaction.

*Bernieri, F. J., Gillis, J. S., Davis, J. M., \& Grahe, J. E. (1996). Dyad rapport and the accuracy of its judgment across situations: A lens model analysis. Journal of Personality and Social Psychology, 71, 110-129.

Birdwhistell, R. L. (1970). Kinesics and context. Philadelphia: University of Pennsylvania Press.

*Blumer, C. H. (1984). The influence of therapist style and subject sex on the accuracy of role-playing a previous event. Unpublished doctoral dissertation, Miami University, Oxford, $\mathrm{OH}$.

*Bond, C. F., Jr., Omar, A., Mahmoud, A., \& Bonser, R. N. (1990). Lie detection across cultures. Journal of Nonverbal Behavior, 14, 189-204.

*Bond, M. H., \& Ho, H. Y. (1978). The effect of relative status and the sex composition of a dyad on cognitive responses and non-verbal behavior of Japanese interviewees. Psychologia: An International Journal of Psychology in the Orient, 21, 128-136.

*Bond, M. H., \& Shiraishi, D. (1974). The effect of body lean and status of an interviewer on the non-verbal behavior of Japanese interviewees. International Journal of Psychology, 9, 117-128.

*Boyce, M., \& Buck, R. (1987). Gender and leadership differences in smiles in sorority and fraternity pictures. Storrs: University of Connecticut. (ERIC Document Reproduction Service No. ED294277)

Brennan-Parks, K., Goddard, M., Wilson, A. E., \& Kinnear, L. (1991). Sex differences in smiling measured in a picture taking task. Sex Roles, 24, 375-382.

*Brion, S., King, K., Alexander, B., B'Jork, F. (2001, March). An examination of the relationship between embarrassment and smiles. Poster presented at the annual meeting of the Western Psychological Association, Maui, HI.

Briton, N. J., \& Hall, J. A. (1995a). Beliefs about female and male nonverbal communication. Sex Roles, 32, 79-90.

*Briton, N. J., \& Hall, J. A. (1995b). Gender-based expectancies and observers' judgments of smiling. Journal of Nonverbal Behavior, 19, $49-65$.

Brody, L. R. (1985). Gender differences in emotional development: A review of theories and research. Journal of Personality, 53, 102-149.

Brody, L. R. (1997). Beyond stereotypes: Gender and emotion. Journal of Social Issues, 53, 369-394.

Brody, L. R. (1999). Gender, emotion, and the family. Cambridge, MA: Harvard University
Brody, L. R., \& Hall, J. A. (1993). Gender and emotion. In M. Lewis \& J. M. Haviland (Eds.), Handbook of emotions (pp. 447-460). New York: Guilford Press.

*Brodzinsky, D. M., Barnet, K., \& Aiello, J. R. (1981). Sex of subject and gender identity as factors in humor appreciation. Sex Roles, 7, 561-573.

*Brosius, H.-B., Mundorf, N., \& Staab, J. F. (1991). The depiction of sex roles in American and German magazine advertisements. International Journal of Public Opinion Research, 3, 306-383.

*Brownlow, S., \& Zebrowitz, L. A. (1990). Facial appearance, gender, and credibility in television commercials. Journal of Nonverbal Behavior, 14, 51-60.

Buck, R., Losow, J. I., Murphy, M. M., \& Costanzo, P. (1992). Social facilitation and inhibition of emotional expression and communication. Journal of Personality and Social Psychology, 63, 962-968.

Burgener, S. C., Jirovec, M., Murrell, L., \& Barton, D. (1992). Caregiver and environmental variables related to difficult behaviors in institutionalized, demented elderly persons. Journal of Gerontology, 47, 242-249.

*Burgoon, J. K., \& Aho, L. (1982). Three field experiments on the effects of violations of conversational distance. Communication Monographs, 49, 71-88.

*Burgoon, J. K., Buller, D. B., \& Guerrero, L. K. (1995). Interpersonal deception IX. Effects of social skill and nonverbal communication on deception success and detection accuracy. Journal of Language and Social Psychology, 14, 289-311.

*Burgoon, J. K., \& LePoire, B. A. (1993). Effects of communication expectancies, actual communication, and expectancy disconfirmation on evaluations of communicators and their communication behavior. $\mathrm{Hu}$ man Communication Research, 20, 67-95.

Canary, D. J., \& Dindia, K. (Eds.). (1998). Sex differences and similarities in communication. Mahwah, NJ: Erlbaum.

Canary, D. J., \& Hause, K. S. (1993). Is there any reason to research sex differences in communication? Communication Quarterly, 41, 129-144.

*Cashdan, E. (1998). Smiles, speech, and body posture: How women and men display sociometric status and power. Journal of Nonverbal Behavior, 22, 209-228.

*Chaiken, S. (1979). Communicator physical attractiveness and persuasion. Journal of Personality and Social Psychology, 37, 1387-1397.

*Chaikin, A. L., \& Derlega, V. J. (1978). Nonverbal mediators of expectancy effects in Black and White children. Journal of Applied Social Psychology, 8, 117-125.

*Chaikin, A. L., Sigler, E., \& Derlega, V. J. (1974). Nonverbal mediators of teacher expectancy effects. Journal of Personality and Social Psychology, 30, 144-149.

*Chapell, M. S. (1997). Frequency of public smiling across the life span. Perceptual and Motor Skills, 85, 1326.

${ }^{*}$ Cherulnik, P. D. (1979). Sex differences in the expression of emotion in a structured social encounter. Sex Roles, 4, 413-424.

*Cherulnik, P. D., \& Evans, R. D. (1984). Facial expressive behaviors of high self-monitors are less sex-typed. Sex Roles, 11, 435-449.

Chiu, C., Hong, Y., Lam, I. C., Fu, J. H., Ton, J. Y., \& Lee, V. S. (1998). Stereotyping and self-presentation: Effects of gender stereotype activation. Group Processes and Intergroup Relations, 1, 81-96.

*Choe, J.-H., Wilcox, G. B., \& Hardy, A. P. (1986). Facial expression in magazine ads: A cross-cultural comparison. Journalism Quarterly, 63, $122-126$.

Cialdini, R. B., Reno, R. R., \& Kallgren, C. A. (1990). A focus theory of normative conduct. Journal of Personality and Social Psychology, 69, $1015-1026$

*Cody, M. J., Lee, W.-S., \& Chao, E. Y. (1989). Telling lies: Correlates of deception among Chinese. In J. P. Forgas \& J. M. Innes (Eds.), Recent advances in social psychology: An international perspective (pp. 359368). North Holland, the Netherlands: Elsevier Science.

*Cody, M. J., \& O’Hair, D. (1983). Nonverbal communication and decep- 
tion: Differences in deception cues due to gender and communicator dominance. Communication Monographs, 50, 175-192.

Cohen, J. (1977). Statistical power analysis for the behavioral sciences (Rev. ed.). New York: Academic Press.

*Coker, D. A., \& Burgoon, J. K. (1987). The nature of conversational involvement and nonverbal encoding patterns. Human Communication Research, 13, 463-494.

Cole, P. M. (1985). Display rules and the socialization of affective displays. In G. Zivin (Ed.), The development of expressive behavior: Biology-environment interactions (pp. 269-290). Orlando, FL: Academic Press.

Cooper, H. M. (1989). Integrating research: A guide for literature reviews (2nd ed.). Newbury Park, CA: Sage.

Darwin, C. (1965). The expression of the emotions in man and animals. New York: Appleton. (Original work published 1872)

*Davis, E. M. (1983). Effects of self-monitoring, need for social approval, and sex on the occurrence of constructive humor. Unpublished doctoral dissertation, University of Nebraska, Lincoln.

Davis, M. (1972). Understanding body movement: An annotated biography. New York: Arno Press.

Davis, M., \& Skupien, J. (Eds.). (1982). Body movement and nonverbal communication: An annotated bibliography, 1971-1980. Bloomington: Indiana University Press.

*Davis, M., \& Weitz, S. (1981). Sex differences in body movements and positions. In C. Mayo \& N. M. Henley (Eds.), Gender and nonverbal behavior (pp. 81-92). New York: Springer.

Deaux, K., \& Major, B. (1987). Putting gender into context: An interactive model of gender-related behavior. Psychological Review, 94, 369-389.

*DeBlasio, C. N., \& Ellyson, S. L. (1994, March). Smiling as a strategy for women in the display of power. Paper presented at the annual meeting of the Eastern Psychological Association, Boston.

*Deckers, L., \& Edington, J. (1979, May). Facial expressions of Mirth as a log-log function of the degree of incongruity in a psychophysical task. Paper presented at the 51st Annual Meeting of the Midwestern Psychological Association, Chicago.

*Deckers, L., Kuhlhorst, L., \& Freeland, L. (1987). The effects of spontaneous and voluntary facial reactions on surprise and humor. Motivation and Emotion, 11, 403-412.

*Deckers, L., \& Salais, D. (1983). Humor as a negatively accelerated function of the degree of incongruity. Motivation and Emotion, 7 , 357-363.

*DeGroot, T., \& Motowidlo, S. J. (1999). Why visual and vocal interview cues can affect interviewers' judgments and predict job performance. Journal of Applied Psychology, 84, 986-993.

*Deleanu, M. J. (1983). The role of aggression and conflict about aggression on humor appreciation. Unpublished doctoral dissertation, University of Tennessee, Knoxville.

*Denmark, F. L. (1977). Styles of leadership. Psychology of Women Quarterly, 2, 99-113.

*Derlega, V. J., McAnulty, M., \& Strout, S. (1980). Pygmalion effects among Blacks: When and how expectancies occur. Journal of Applied Social Psychology, 10, 260-271.

*DeSantis, M., \& Sierra, N. (2000). Women smiled more often and openly than men when photographed for a pleasant, public occasion in 20super(th) century United States society. Psychology: A Quarterly Journal of Human Behavior, 37, 21-31.

DePaulo, B. M. (1992). Nonverbal behavior and self presentation. Psychological Bulletin, 111, 203-243.

*Deutsch, F. M. (1990). Status, sex, and smiling: The effect of role on smiling in men and women. Personality and Social Psychology Bulletin, 16, 531-540.

Deutsch, F. M., LeBaron, D., \& Fryer, M. (1987). What is in a smile? Psychology of Women Quarterly, 11, 341-352.
Dindia, K., \& Allen, A. (1992). Sex differences in self-disclosure: A meta-analysis. Psychological Bulletin, 112, 106-124.

*Dodd, D. K., Russell, B. L., \& Jenkins, C. (1999). Smiling in school yearbook photos: Gender differences from kindergarten to adulthood. Psychological Record, 49, 543-554.

*Dovidio, J. F., Brown, C. E., Heltman, K., Ellyson, S. L., \& Keating, C. F. (1988). Power displays between women and men in discussions of gender-linked tasks: A multichannel study. Journal of Personality and Social Psychology, 55, 580-587.

*Drake, E. (1987). Self disclosure, trust building exercises, and non-verbal communication. Unpublished honors' thesis, Skidmore College, Saratoga Springs, NY.

Eagly, A. H. (1986). Some meta-analytic approaches to examining the validity of gender-difference research. In J. S. Hyde \& M. C. Linn (Eds.), The psychology of gender: Advances through meta-analysis. Baltimore: Johns Hopkins University Press.

Eagly, A. H. (1987). Sex differences in social behavior: A social role interpretation. Hillsdale, NJ: Erlbaum .

Eagly, A. H., \& Carli, L. L. (1981). Sex of researchers and sex-typed communications as detriments of sex differences in influenceability: A meta-analysis of social influence studies. Psychological Bulletin, 90 $1-20$.

Eagly, A. H., Chen, S., Chaiken, S., \& Shaw-Barnes, K. (1999). The impact of attitudes on memory: An affair to remember. Psychological Bulletin, $125,64-89$

Eagly, A. H., Karau, S. J., \& Makhijani, M. (1995). Gender and the effectiveness of leaders: A meta-analysis. Psychological Bulletin, 117 125-145.

Eakins, B. W., \& Eakins, R. G. (1978). Sex differences in human communication. Boston: Houghton Mifflin.

Eccles, J. S., \& Bryan, J. (1994). Adolescence and gender-role transcendence. In M. Stevenson (Ed.), Gender roles across the life span (pp. 111-148). Muncie, IN: Ball State University Press.

*Edelmann, R. J. (1982). The effect of embarrassed reactions upon others. Australian Journal of Psychology, 34, 359-367.

Edelmann, R. J., Asendorpf, J., Contarello, A., \& Zammuner, V. (1989). Self-reported expression of embarrassment in five European cultures. Journal of Cross-Cultural Psychology, 20, 357-371.

*Edelmann, R. J., \& Hampson, S. E. (1981). Embarrassment in dyadic interaction. Social Behavior and Personality, 9, 171-177.

Eibl-Eibesfeldt, I. (1989). Human ethology. New York: Aldine de Gruyter. Ekman, P. (1985). Telling lies. New York: Norton.

Ekman, P. (1994). Strong evidence for universals in facial expressions: A reply to Russell's mistaken critique. Psychological Bulletin, 115, 268287

Ekman, P., Davidson, R. J., \& Friesen, W. V. (1990). The Duchenne smile: Emotional expression and brain physiology: II. Journal of Personality and Social Psychology, 58, 342-353.

Ekman, P., \& Friesen, W. V. (1975). Unmasking the face: A guide to recognizing emotions from facial cues. Englewood Cliffs, NJ: PrenticeHall

Ekman, P., \& Friesen, W. V. (1978). Facial action coding system (FACS): A technique for the measurement of facial action. Palo Alto, CA: Consulting Psychologists Press.

Ekman, P., \& Friesen, W. V. (1982). Felt, false, and miserable smiles. Journal of Nonverbal Behavior, 6, 238-252.

Ekman, P., Friesen, W. V., \& Ancoli, S. (1980). Facial signs of emotional experience. Journal of Personality and Social Psychology, 39, 11251134.

Elman, D., Schulte, D. C., \& Bukoff, A. (1977). Effects of facial expression and stare duration on walking speed: Two field experiments. Environmental Psychology and Nonverbal Behavior, 2, 93-99.

*Fernandez-Dols, J.-M., \& Ruiz-Belda, M.-A. (1995). Are smiles a sign of 
happiness? Gold medal winners at the Olympic Games. Journal of Personality and Social Psychology, 69, 1113-1119.

*Fernandez-Dols, J. M., \& Ruiz-Belda, M.-A. (1997). Spontaneous facial behavior during intense emotional episodes: Artistic truth and optical truth. In J. A. Russell \& J. M. Fernandez-Dols (Eds.), The psychology of facial expression: Vol. 2. Studies in emotion and social interaction (pp. 255-274). Cambridge, England: Cambridge University Press.

*Field, T. (1978). Interaction behaviors of primary versus secondary caretaker fathers. Developmental Psychology, 14, 183-184.

*Field, T. (1981). Fathers' interaction with their high-risk infants. Infant Mental Health Journal, 2, 249-256.

*Field, T., Vega-Lahr, N., Goldstein, S., \& Scafidi, F. (1987). Interaction behavior of infants and their dual-career parents. Infant Behavior and Development, 10, 371-377.

*Finkelstein, S. (1978). The relationship between physical attractiveness an nonverbal behaviors. Unpublished honors' thesis, Hampshire College, Amherst, MA.

Fischer, A. H. (1993). Sex differences in emotionality: Fact or stereotype? Feminism and Psychology, 3, 303-318.

Fischer, A. H. (Ed.). (2000). Gender and emotion: Social psychological perspectives. Cambridge, England: Cambridge University Press.

Fischer, A. H. (2002, July). Anger politics: Why women are bitches. In S. Shields (Chair), Taking sides: The politics of emotion in everyday life. Symposium conducted at the meeting of the International Society for Research on Emotion, Cuenca, Spain.

Fiske, S. T., \& Stevens, L. E. (1993). What's so special about sex? Gender stereotyping and discrimination. In S. Oskamp \& M. Costanzo (Eds.), Gender issues in contemporary society (pp. 173-196). Newbury Park, CA: Sage.

*Frances, S. J. (1979). Sex differences in nonverbal behavior. Sex Roles, 5 , 519-535.

*Freedman, D. G. (1979). Human sociobiology: A holistic approach. New York: Free Press.

Fridlund, A. J. (1991). Sociality of solitary smiling: Potentiation by an implicit audience. Journal of Personality and Social Psychology, 60, 229-240.

Fridlund, A. J. (1994). Human facial expression: An evolutionary view. San Diego, CA: Academic Press.

Fridlund, A. J., Sabini, J. P., Hedlund, L. E., Schaut, J. A., Shenker, J. I., \& Knauer, M. J. (1990). Audience effects on solitary faces during imagery: Displaying to the people in your head. Journal of Nonverbal Behavior, 142, 113-137.

*Friedman, H. S., DiMatteo, M. R., \& Mertz, T. I. (1980). Nonverbal communication on television news: The facial expression of broadcasters during coverage of a presidential election campaign. Personality and Social Psychology Bulletin, 6, 427-435.

*Friedman, H. S., \& Miller-Herringer, T. (1991). Nonverbal display of emotion in public and private: Self-monitoring, personality, and expressive cues. Journal of Personality and Social Psychology, 61, 766-775.

*Frisch, I. (1995). Facial activities of women and men in dyadic interactions with a partner of the same sex. Zeitschrift Fuer Differentielle und Diagnostische Psychologie, 16, 33-42.

Frith, H., \& Kitzinger, C. (1998). "Emotion work" as a participant resource: A feminist analysis of young women's talk-in-interaction. Sociology, 32, 299-320.

*Fry, A. M., \& Willis, F. N. (1971). Invasion of personal space as a function of the age of the invader. Psychological Record, 21, 385-389.

Frymier, A. B., Klopf, D. W., \& Ishii, S. (1990). Japanese and Americans compared on the affect orientation construct. Psychological Reports, 66, 985-986.

Fujita, B. N., Harper, R. G., \& Wiens, A. H. (1980). Encoding-decoding of nonverbal emotional messages: Sex differences in spontaneous and enacted expressions. Journal of Nonverbal Behavior, 4, 131-145.

*Fuller, R. G. C., \& Sheehy-Skeffington, A. (1974). Effects of group laughter on responses to humorous material, a replication and extension. Psychological Reports, 35, 531-534.

*Gallois, C., \& Callan, V. J. (1988). Communication accommodation and the prototypical speaker: Predicting evaluations of status and solidarity. Language \& Communication, 8, 271-283.

*Garcia, S., Stinson, L., Ickes, W., \& Bissonnette, V. (1991). Shyness and physical attractiveness in mixed-sex dyads. Journal of Personality and Social Psychology, 61, 35-49.

*Gavinski, I. (1986). Differential sensitivity of humor ratings and mirth responses to cognitive and affective components of the humor response. Journal of Personality and Social Psychology, 51, 209-214.

*Gerber, W. S., \& Routh, D. K. (1975). Humor response as related to violation of expectancies and to stimulus intensity in a weight-judgment task. Perceptual and Motor Skills, 41, 673-674.

*Gifford, R. (1994). A lens-mapping framework for understanding the encoding and decoding of interpersonal dispositions in nonverbal behavior. Journal of Personality and Social Psychology, 66, 115-132.

Goffman, E. (1979). Gender advertisements. New York: Harper \& Row.

Goldenthal, P., Johnston, R. E., \& Kraut, R. E. (1981). Smiling, appeasement, and the silent bared-teeth display. Ethology and Sociobiology, 2, $127-133$.

*Gottman, J. M., \& Levenson, R. W. (1992). Marital processes predictive of later dissolution: Behavior, physiology, and health. Journal of Personality and Social Psychology, 63, 221-233.

*Guerrero, L. K. (1997). Nonverbal involvement across interactions with same-sex friends, opposite-sex friends and romantic partners: Consistency or change. Journal of Social \& Personal Relationships, 14, 31-58.

*Halberstadt, A. G. (1983). Gender and nonverbal behavior: Of relevance and rigor. Semiotica, 45, 351-369.

*Halberstadt, A. G., Dovidio, J. F., \& Davidson, L. A. (1988, October). Power, gender, and smiling. Paper presented at the annual meeting of the Society of Experimental Social Psychology, Madison, WI.

*Halberstadt, A. G., Hayes, C. W., \& Pike, K. M. (1988). Gender and gender role differences in smiling and communication consistency. Sex Roles, 19, 589-604.

*Halberstadt, A. G., \& Saitta, M. B. (1987). Gender, nonverbal behavior, and perceived dominance: A test of theory. Journal of Personality and Social Psychology, 53, 257-272.

Hall, E. J. (1993). Smiling, deferring, and flirting: Doing gender by giving "good service." Work and Occupations, 20, 452-473.

Hall, J. A. (1984). Nonverbal sex differences: Communication accuracy and expressive style. Baltimore: Johns Hopkins University Press.

Hall, J. A. (1987). On explaining gender differences: The case of nonverbal communication. In P. Shaver \& C. Hendrick (Eds.), Sex and gender (pp. 177-200). Newbury Park, CA: Sage.

*Hall, J. A. (1992). [Physician and patient communication]. Unpublished raw data.

Hall, J. A. (1998). How big are nonverbal sex differences? The case of smiling and sensitivity to nonverbal cues. In D. J. Canary \& K. Dindia (Eds.), Sex differences and similarities in communication: Critical essays and empirical investigations of sex and gender in interaction (pp. 155-177). Mahwah, NJ: Erlbaum.

Hall, J. A., \& Briton, N. J. (1993). Gender, nonverbal behavior, and expectations. In P. D. Blanck (Ed.), Interpersonal expectations: Theory, research, and applications (pp. 276-295). Cambridge, England: Cambridge University Press.

Hall, J. A., Carter, J. D., \& Horgan, T. G. (2000). Gender differences in nonverbal communication of emotion. In A. H. Fischer (Ed.), Gender and emotion: Social psychological perspectives (pp. 97-117). Cambridge, England: Cambridge University Press.

*Hall, J. A., Carter, J. D., Jiminez, M. C., Frost, N. A., \& Smith LeBeau, L. B. (2002). Smiling and relative status in news photographs. Journal of Social Psychology, 142, 500-510. 
*Hall, J. A., \& Friedman, G. B. (1999). Status, gender, and nonverbal behavior: A study of structured interactions between employees of a company. Personality and Social Psychology Bulletin, 25, 1082-1091.

Hall, J. A., \& Halberstadt, A. G. (1986). Smiling and gazing. In J. S. Hyde \& M. C. Inn (Eds.), The psychology of gender: Advances through meta-analysis (pp. 136-185). Baltimore: Johns Hopkins University Press.

*Hall, J. A., Horgan, T. G., \& Carter, J. D. (2002). Assigned and felt status in relation to observer-coded and participant-reported smiling. Journal of Nonverbal Behavior, 26, 63-81.

*Hall, J. A., LeBeau, L. S., Reinoso, J. G., \& Thayer, F. (2001). Status, gender, and nonverbal behavior in candid and posed photographs: A study of conversations between university employees. Sex Roles, 44, 677-692.

*Hall, J. A., \& Rosenthal, R. (1991). Testing for moderator variables in meta-analysis: Issues and methods. Communication Monographs, 58, 437-448.

*Harrigan, J. A., \& O'Connell, D. M. (1996). How do you look when feeling anxious? Facial displays of anxiety. Personality \& Individual Differences, 21, 205-212.

*Harrigan, J. A., Oxman, T. E., \& Rosenthal, R. (1985). Rapport expressed through nonverbal behavior. Journal of Nonverbal Behavior, 9, 95-110.

*Harris, C., \& Alvarado, N. (2000, June). Gender differences in response to emotional states. Paper presented at the annual meeting of the American Psychological Society, Toronto, Ontario, Canada.

Harris, M. J., \& Rosenthal, R. (1985). Mediation of interpersonal expectancy effects: 31 meta-analyses. Psychological Bulletin, 97, 363-386.

*Hecht, M. A., \& LaFrance, M. (1998). License or obligation to smile: Power, sex and smiling. Personality and Social Psychology Bulletin, 24, 1326-1336.

*Hecht, M. A., LaFrance, M., \& Allard, L. (1994). Smiling and status in mixed-sex interactions. Unpublished manuscript, Boston College, Chestnut Hill, MA

Hedges, L. V. (1981). Distribution theory for Glass's estimator of effect size and related estimators. Journal of Educational Statistics, 6, 107128.

Hedges, L. V. (1994). Fixed effects models. In H. Cooper \& L. V. Hedges (Eds.), The handbook of research synthesis (pp. 285-299). New York: Russell Sage Foundation.

Hedges, L. V., \& Olkin, I. (1980). Vote-counting methods in research synthesis. Psychological Bulletin, 88, 359-369.

*Hemsley, G. D. (1977). Experimental studies in the behavioral indicants of deception. Unpublished doctoral dissertations, University of Toronto, Toronto, Ontario, Canada.

*Henley, N. M. (1977). Body politics: Power, sex, and nonverbal communication. Englewood Cliffs, NJ: Prentice-Hall.

*Hersh, J. B. (1971). Effects of referral information on testers. Journal of Consulting and Clinical Psychology, 37, 116-122.

Hess, U., Senecal, S., Kirouac, G., Herrera, P., Philippot, P., \& Kleck, R. E. (2000). Emotional expressivity in men and women: Stereotypes and self-perceptions. Cognition \& Emotion, 14, 609-642.

*Hinsz, V. B., \& Tomhave, J. A. (1991). Smile and (half) the world smiles with you, frown and you frown alone. Personality and Social Psychology Bulletin, 17, 586-592.

*Ho, R., \& Mitchell, S. (1982). Students' nonverbal reaction to tutors' warm/cold nonverbal behavior. Journal of Social Psychology, 118, 121130.

Hochschild, A. (1983). The managed heart: Commercialization of human feeling. Berkeley: University of California Press.

*Honeycutt, J. M. (1989). Effects of preinteraction expectancies on interaction involvement and behavioral responses in initial interaction. Journal of Nonverbal Behavior, 13, 25-36.

*Ickes, W. (1984). Compositions in Black and White: Determinants of interaction in interracial dyads. Journal of Personality and Social Psychology, 47, 330-341.

*Ickes, W., \& Barnes, R. D. (1978). Boys and girls together-and alienated: On enacting stereotyped sex roles in mixed-sex dyads. Journal of Personality and Social Psychology, 36, 669-683.

Ickes, W., Patterson, M. L., Rajecki, D. W., \& Tanford, S. (1982). Behavioral and cognitive consequences of reciprocal versus compensatory responses to preinteraction expectancies. Social Cognition, 1, 160-190.

*Ickes, W., Robertson, E., Tooke, W., \& Teng, G. (1986). Naturalistic social cognition: Methodology, assessment, and validation. Journal of Personality and Social Psychology, 51, 66-82.

*Ickes, W., Schermer, B., \& Steeno, J. (1979). Sex and sex-role influences in same-sex dyads. Social Psychology Quarterly, 42, 373-385.

*Ickes, W., Tooke, W., Stinson, L., Baker, V. L., \& Bissonnette, V. (1988). Naturalistic social cognition: Intersubjectivity in same-sex dyads. Journal of Nonverbal Behavior, 12, 58-84.

*Ickes, W., \& Turner, M. (1983). On the social advantages of having an older, opposite-sex sibling: Birth order influences in mixed-sex dyads. Journal of Personality and Social Psychology, 45, 210-222.

Izard, C. E. (1997). Emotions and facial expressions: A perspective from differential emotions theory. In J. A. Russell \& J. M. Fernandez-Dols (Eds.), The psychology of facial expression: Vol. 2. Studies in emotion and social interaction (pp. 57-77). Cambridge, England: Cambridge University Press.

Johnson, B. T., \& Eagly, A. H. (2000). Quantitative synthesis of social psychological research. In H. Reis \& C. Judd (Eds.), Handbook of research methods in social and personality psychology (pp. 496-528). New York: Cambridge University Press.

*Johnson, C. (1994). Gender, legitimate authority, and leader-subordinate conversations. American Sociological Review, 59, 122-135.

*Johnson, C. B. (1989). Bias in the selection interview: Self- or otherdirected? Unpublished doctoral dissertation, Kansas State University, Manhattan.

*Jones, E., Gallois, C., Callan, V., \& Barker, M. (1999). Strategies of accommodation: Development of a coding system for conversational interaction. Journal of Language \& Social Psychology, 18, 123-152.

*Jorgenson, D. O. (1978). Nonverbal assessment of attitudinal affect with the smile-return technique. Journal of Social Psychology, 106, 173-179.

Joshi, M. S., \& Maclean, M. (1994). Indian and English children's understanding of the distinction between real and apparent emotion. Child Development, 65, 1372-1384.

*Kahlbaugh, P. E., \& Haviland, J. M. (1994). Nonverbal communication between parents and adolescents: A study of approach and avoidance behaviors. Journal of Nonverbal Behavior, 18, 91-113.

Keating, C. F. (1985). Human dominance signals: The primate in us. In S. L. Ellyson \& J. F. Dovidio (Eds.), Power, dominance and nonverbal behavior (pp. 89-108). New York: Springer.

*Keller, E. (1980). Effects of physical attractiveness of infants on parental behavior. Unpublished doctoral dissertation, California School of Professional Psychology, Berkeley.

*Keltner, D. (1997). Signs of appeasement: Evidence for the distinct displays of embarrassment, amusement, and shame. Journal of Personality and Social Psychology, 68, 441-454.

*Keltner, D., \& Bonanno, G. A. (1997). A study of laughter and dissociation: Distinct correlates of laughter and smiling during bereavement. Journal of Personality and Social Psychology, 73, 687-702.

*Kendon, A., \& Ferber, A. (1973). A description of some human greetings. In R. P. Michael \& J. H. Crook (Eds.), Comparative ecology and behaviour of primates (pp. 591-668). London: Academic Press.

*Kennedy, C. W., \& Camden, C. (1983). Interruptions and nonverbal gender differences. Journal of Nonverbal Behavior, 8, 91-108.

*Kenny, D. A., Horner, C., Kashy, D. A., \& Chu, L. (1992). Consensus at zero acquaintance: Replication, behavioral cues, and stability. Journal of Personality and Social Psychology, 62, 88-97. 
Kenny, D. A. \& la Voie, L. (1985). Separating individual and group effects. Journal of Personality and Social Psychology, 48, 339-348.

Kierstead, D., D’Agostino, P., \& Dill, H. (1988). Sex role stereotyping of college professors: Bias in students' ratings of instructors. Journal of Educational Psychology, 80, 342-244.

Kleck, R. E., Vaughan, R. C., Cartwright-Smith, H., Vaughan, K. B., Colby, C. A., \& Lanzetta, J. T. (1976). Effects of being observed on expressive, subjective, and physiological responses to painful stimuli. Journal of Personality and Social Psychology, 34, 1211-1218.

Knapp, M. L., \& Hall, J. A. (1997). Nonverbal communication in human interaction (4th ed.). New York: Harcourt Brace Jovanovich.

Knight, G. P., Fabes, R A., \& Higgins, D. A. (1996). Concerns about drawing causal inferences from meta-analyses: An example in the study of gender differences in aggression. Psychological Bulletin, 119, 410421.

*Kolaric, G. C., \& Galambos, N. L. (1995). Face-to-face interactions in unacquainted female-male adolescent dyads: How do girls and boys behave? Journal of Early Adolescence, 15, 363-382.

*Kollar, E. K. (1986). Physiological, sex and personality variables related to facial expression of emotions. Unpublished doctoral dissertation, Catholic University of America, Washington, DC.

*Koo, J., Ambady, N., \& Hecht, M. A. (1997, June). Verbal and nonverbal communication across power: A cross-cultural study. Paper presented at the annual meeting of the American Psychological Society, Washington, DC

Korzenny, B. A. G., Korzenny, F., \& Sanchez de Rota, G. (1985). Women's communication in Mexican organizations. Sex Roles, 12, 867-876.

Kramer, C. (1977). Perceptions of male and female speech. Language and Speech, 20, 151-161

Kraut, R. E., \& Johnston, R. E. (1979). Social and emotional messages of smiling: An ethological approach. Journal of Personality and Social Psychology, 37, 1539-1553.

Kring, A. M. (2000). Gender and anger. In A. H. Fischer (Ed.), Gender and emotion: Social psychological perspectives (pp. 211-231). Cambridge, England: Cambridge University Press

Kring, A. M., \& Gordon, A. L. (1998). Sex differences in emotion. Journal of Personality and Social Psychology, 74, 686-703.

*LaFrance, M. (1985). Does your smile reveal your status? Social Science News Letter, 70, 15-18.

LaFrance, M. (1997). Pressure to be pleasant: Effects of sex and power on reactions to not smiling. Revue Internationale de Psychology Sociale/ International Review of Social Psychology, 2, 95-108.

LaFrance, M., \& Banaji, M. (1992). Towards a reconsideration of the gender emotion relationship. In M. S. Clark (Ed.), Emotion and social behavior (pp. 178-201). Beverly Hills, CA: Sage.

*LaFrance, M., \& Carmen, B. (1980). The nonverbal display of psychological androgyny. Journal of Personality and Social Psychology, 38, $36-49$.

LaFrance, M., \& Hecht, M. A. (1995). Why smiles generate leniency. Personality and Social Psychology Bulletin, 21, 207-214.

LaFrance, M., \& Hecht, M. A. (1999). Obliged to smile: The effect of power and gender on facial expression. In P. Philippot, R. S. Feldman, \& E. J. Coats (Eds.), The social context of nonverbal behavior (pp. 45-70). Cambridge, England: Cambridge University Press.

LaFrance, M., \& Mayo, C. (1978). Moving bodies: Nonverbal communication in social relationships. Monterey, CA: Brooks/Cole.

*Lamb, M. E., Frodi, A. M., Hwang, C.-P., Frodi, M., \& Steinberg, J. (1982). Mother- and father-infant interaction involving play and holding in traditional and nontraditional Swedish families. Developmental Psychology, 18, 215-221.

Leary, M. R., \& Kowalksi, R. M. (1995). Social anxiety. New York: Guilford Press.

Leary, M. R., Nexlek, J. B., Downs, D., Radford-Davenport, J., Martin, J., \& McMullen, A. (1994). Self-presentation in everyday interactions:
Effects of target familiarity and gender composition. Journal of Personality and Social Psychology, 67, 664-673.

*Lefébvre, L. M. (1973). An experimental approach to the use of ingratiation tactics under homogeneous and heterogeneous dyads. European Journal of Social Psychology, 3, 427-445.

*Leve, R. M., \& Burdick, L. (1977). A functional analysis of reinforcement within a social system. Behavior Therapy, 8, 456-459.

*Leventhal, H., \& Cupchik, G. C. (1975). The informational and facilitative effects of an audience upon expression and the evaluation of humorous stimuli. Journal of Experimental Social Psychology, 11, 363 380 .

*Leventhal, H., \& Mace, W. (1970). The effect of laughter on evaluation of a slapstick movie. Journal of Personality, 38, 16-30.

*Lippa, R., \& Connelly, S. (1991). Gender diagnosticity: A new Bayesian approach to gender-related individual differences. Journal of Personality and Social Psychology, 59, 1051-1065.

*Lochman, J. E., \& Allen, G. (1981). Nonverbal communication of couples in conflict. Journal of Research in Personality, 15, 253-269.

* Mackey, W. C. (1976). Parameters of the smile as a social signal. Journal of Genetic Psychology, 129, 125-130.

Manstead, A. S. R. (1991). Expressiveness as an individual difference. In R. S. Feldman \& B. Rime (Eds.), Fundamentals of nonverbal behavior (pp. 285-328). Cambridge, England: Cambridge University Press.

*Martin, G. N., \& Gray, C. D. (1996). The effects of audience laughter on men's and women's responses to humor. Journal of Social Psychology, 136, 221-231.

*Massé, M. A., \& Rosenblum, K. (1988). Male and female created they them: The depiction of gender in the advertising of traditional women's and men's magazines. Women's Studies International Forum, 11, 127144 .

Masters, R. D., Sullivan, D. G., Lanzetta, J. T., McHugo, G. J., \& Englis, B. G. (1985). The facial displays of leaders: Toward an ethology of human politics. Journal of Social and Biological Structures, 9, 319-343.

Matlin, M. M. (1993). The psychology of women (2nd ed.). New York: Harcourt Brace.

Matsumoto, D., \& Ekman, P. (1989). American/Japanese cultural differences in intensity ratings of facial expressions of emotion. Motivation \& Emotion, 13, 143-157.

*McAdams, D. P., Jackson, R. J., \& Kirshnit, C. (1984). Looking, laughing, and smiling in dyads as a function of intimacy motivation and reciprocity. Journal of Personality, 52, 261-273.

*McClintock, C. C., \& Hunt, R. G. (1975). Nonverbal indicators of affect and deception in an interview setting. Journal of Applied Social Psychology, 5, 54-67.

*McCormick, N. B., \& Jones, A. J. (1989). Gender differences in nonverbal flirtation. Journal of Sex Education and Therapy, 15, 271-282.

*McLean, J. D. (1971). Sex-correlated differences in human smiling behavior: A preliminary investigation. Unpublished manuscript, University of Chicago.

*Mehrabian, A. (1971). Nonverbal betrayal of feeling. Journal of Experimental Research in Personality, 5, 64-73.

*Mehrabian, A., \& Williams, M. (1969). Nonverbal concomitants of perceived and intended persuasiveness. Journal of Personality and Social Psychology, 13, 37-58.

Melville, H. (1949). Pierre: Or the Ambiguities. New York: Hendricks House. (Original work published 1852)

*Messina, S. L. (1977). Sexual differences in smiling behavior. Unpublished master's thesis, University of South Dakota, Fargo.

*Mills, J. (1984). Self-posed behaviors of females and males in photographs. Sex Roles, 10, 633-637.

Mischel, W. (1977). The interaction of person and situation. In D. Magnusson \& N. S. Endler (Eds.), Personality at the crossroads: Current issues in interactional psychology (pp. 333-352). Hillsdale, NJ: Erlbaum. 
*Morse, C. (1982). College yearbook pictures: More females smile than males. Journal of Psychology, 110, 3-6.

Moskowitz, D. S., Suh, E. J., \& Desaulniers, J. (1994). Situational influences on gender differences in agency and communion. Journal of Personality and Social Psychology, 8, 249-270.

Mullen, B., Futrell, D. E., Stairs, D., Tice, D. M., Baumeister, R. F., Dawson, K. E., et al. (1986). Newscasters' facial expressions and voting behavior of viewers: Can a smile elect a president? Journal of Personality and Social Psychology, 51, 291-295.

*Myers, B. J. (1980). An intervention to improve parent-to-infant bonding in mothers and fathers of newborns. Unpublished doctoral dissertation, Temple University, Philadelphia.

*Myszka, T. J. (1968). Situational and interpersonal determinants of eye contact, direction of gaze aversion, smiling and other nonverbal behaviors during an interview. Unpublished doctoral dissertation, University of Windsor, Windsor, Ontario, Canada.

*Noller, P. (1985). Negative communications in marriage. Journal of Social and Personal Relationships, 2, 289-301.

*Noller, P., \& Callan, V. J. (1989). Nonverbal behavior in families with adolescents. Journal of Nonverbal Behavior, 13, 47-64.

*Noller, P., \& Gallois, C. (1986). Sending emotional messages in marriage: Non-verbal behaviour, sex, and communication clarity. British Journal of Social Psychology, 25, 287-297.

Obudho, C. E. (1979). Human nonverbal behavior : An annotated bibliography. Westport, CT: Greenwood Press.

Ochanomizu, U. (1991). Representation forming in Kusyo behavior. Japanese Journal of Developmental Psychology, 2, 25-31.

*O'Quin, K., \& Aronoff, J. (1981). Humor as a technique of social influence. Social Psychology Quarterly, 44, 349-357.

*Otta, E. (1998). Sex differences over age groups in self-posed smiling photographs. Psychological Reports, 83, 907-913.

*Parke, R. D., Grossman, K., \& Tinsley, B. R. (1981). Father-motherinfant interaction in the newborn period: A German-American comparison. In T. M. Field, A. M. Sostek, P. Vietze, \& P. H. Leiderman (Eds.), Culture and early interactions (pp. 95-113). Hillsdale, NJ: Erlbaum.

*Parke, R. D., \& O'Leary, S. E. (1976). Family interaction in the newborn period: Some findings, some observations, and some unresolved issues. In K. F. Riegel \& J. A. Meacham (Eds.), The developing individual in a changing world (pp. 653-663). The Hague, the Netherlands: Mouton.

*Parke, R. D., O'Leary, S. E., \& West, S. (1972). Mother-father-newborn interaction: Effects of maternal medication, labor, and sex of infant. Proceedings of the Annual Convention of the American Psychological Association, 7, 85-86.

*Parke, R. D., \& Sawin, D. B. (1980). The family in early infancy: Social interactional and attitudinal analyses. In F. A. Pedersen (Ed.), The father-infant relationship: Observational studies in the family setting (pp. 44-70). New York: Praeger.

*Pasupathi, M., Carstensen, L. L., Levenson, R. W., \& Gottman, J. M. (1999). Responsive listening in long-married couples: A psycholinguistic perspective. Journal of Nonverbal Behavior, 23, 173-193.

Patterson, M. L., Reidhead, S. M., Gooch, M. V., \& Stopka, S. J. (1984). A content-classified bibliography of research on the immediacy behaviors: 1965-82. Journal of Nonverbal Behavior, 8, 360-393.

*Pedersen, F. A., Anderson, B. J., \& Cain, R. L., Jr. (1980). Parent-infant and husband-wife interactions observed at age 5 months. In F. A. Pedersen (Ed.), The father-infant relationship: Observational studies in the family setting (pp. 71-86). New York: Praeger.

*Pedersen, F. A., Suwalsky, J. T. D., Cain, R. L., Zaslow, M. J., \& Rabinovich, B. A. (1987). Paternal care of infants during maternal separations: Associations with father-infant interaction at one year. Psychiatry, 50, 193-205.

Perry, L. A. M., Turner, L. H., \& Sterk, H. M. (1992). (Eds.). Constructing and reconstructing gender: The links among communication, gender, and language. Albany: State University of New York Press.

*Pilkonis, P. A. (1977). The behavioral consequences of shyness. Journal of Personality, 45, 596-611.

Philippot, P., Feldman, R. S., \& Coats, E. J. (Eds.). (1999). The social context of nonverbal behavior. Cambridge, England: Cambridge University Press.

*Prkachin, K. M., \& Mercer, S. R. (1989). Pain expression in patients with shoulder pathology: Validity, properties and relationship to sickness impact. Pain, 39, 257-265.

Pruett, K., \& Litzenberger, B. (1992). Latency development in children of primary nurturing fathers: Eight year follow-up. Psychoanalytic Study of the Child, 47, 85-101.

*Putnam, L. L., \& McCallister, L. (1980). Situational effects of task and gender on nonverbal display. In D. Nimmo (Ed.), Communication yearbook (Vol. 4, pp. 679-697). New Brunswick, NJ: Transaction Books.

* Ragan, J. M. (1982). Gender displays in portrait photographs. Sex Roles, 8, 33-43.

Reid, P. T., \& Trotter, K. (1993). Children's self-presentations with infants: Gender and ethnic comparisons. Sex Roles, 29, 171-181.

*Ricci Bitti, P., Giovannini, D., \& Palmonari, A. (1974). A study of the interviewer effect in two person interaction. Italian Journal of Psychology, 1, 305-315.

*Riggio, R. E., \& Friedman, H. S. (1983). Individual differences and cues to deception. Journal of Personality and Social Psychology, 45, 899915.

*Riggio, R. E., Tucker, J., \& Widaman, K. F. (1987). Verbal and nonverbal cues as mediators of deception ability. Journal of Nonverbal Behavior, 11, 126-145.

Risman, B. (1987). Intimate relationships from a microstructural perspective. Gender and Society, 1, 6-32.

*Roopnarine, J. L., Talukder, E., Jain, D., Joshi, P., \& Srivastav, P. (1990). Characteristics of holding, patterns of play, and social behaviors between parents and infants in New Delhi, India. Developmental Psychology, 26, 667-673.

*Rose, S. M. (1972). Sex-linked status differentiation in smiling. Unpublished manuscript, University of Chicago.

*Rosenblatt, P. C. (1974). Behavior in public places: Comparison of couples accompanied and unaccompanied by children. Journal of Marriage and the Family, 36, 750-755.

*Rosenblatt, P. C., \& Cleaves, W. T. (1981). Family behavior in public places: Interaction patterns within and across generations. MerrillPalmer Quarterly, 27, 257-269.

*Rosenfeld, H. M. (1966). Approval-seeking and approval-inducing functions of verbal and nonverbal responses in the dyad. Journal of Personality and Social Psychology, 4, 597-605.

*Rosenthal, R. (1976). Experimenter effects in behavioral research. New York: Irvington.

Rosenthal, R. (1979). The "file drawer problem" and tolerance for null results. Psychological Bulletin, 85, 638-641.

Rosenthal, R. (1991). Meta-analytic procedures for social research (Rev. ed.). Newbury Park, CA: Sage.

Rosenthal, R., \& Rosnow, R. L. (1991). Essentials of behavioral research: Methods and data-analysis. New York: McGraw Hill.

Rosenthal, R., \& Rubin, D. B. (1986). Meta-analytic procedures for combining studies with multiple effect sizes. Psychological Bulletin, 99, 400-406.

Russell, J. A., \& Fernandez-Dols, J. M. (Eds.). (1997). The psychology of facial expression: Vol. 2. Studies in emotion and social interaction. Cambridge, England: Cambridge University Press.

Rutherford, S. (2001). Any differences? An analysis of gender management styles in a large airline. Gender, Work and Organization, 8, $326-435$.

Saarni, C. (1993). Socialization of emotion. In M. Lewis \& J. M. Haviland 
(Eds.), Handbook of emotions (pp. 435-446). New York: Guilford Press.

Saarni, C., \& Weber, H. (1999). Emotional displays and dissemblance in childhood: Implications for self-presentation. In P. Philippot, R. S. Feldman, \& E. J. Coats (Eds.), The social context of nonverbal behavior (pp. 71-105). Cambridge, England: Cambridge University Press.

*Sarason, I. G., \& Winkel, G. H. (1966). Individual differences among subjects and experimenters and subjects' self-descriptions. Journal of Personality and Social Psychology, 3, 448-457.

*Scarlett, H. H. (1978). Communication exchange behaviors of men and women from middle and lower socioeconomic class backgrounds. Unpublished doctoral dissertation, Clark University, Worcester, MA.

*Scherer, K. R., \& Ceschi, G. (2000). Criteria for emotion recognition from verbal and nonverbal expression: Studying baggage loss in the airport. Personality and Social Psychology Bulletin, 26, 327-339.

*Schleidt, M., Schiefenhövel, W., Stanjek, K., \& Krell, R. (1980). "Caring for a baby" behavior: Reactions of passersby to a mother and baby. Man-Environment Systems, 10, 73-82.

*Segrin, C. (1992). Specifying the nature of social skill deficits associated with depression. Human Communication Research, 19, 89-123.

*Shotland, R. L., \& Craig, J. M. (1988). Can men and women differentiate between friendly and sexually interested behavior? Social Psychology Quarterly, 51, 66-73.

*Simmons, J. M. (1986). Gender differences of nonverbal power cues in television cues. Paper presented at the annual conference of the American Academy of Advertising, Norman, OK.

*Simpson, J. A., Gangestad, S. W., \& Biek, M. (1993). Personality and nonverbal social behavior: An ethological perspective of relationship initiation. Journal of Experimental Social Psychology, 29, 434461

*Smith, A. (1983). Nonverbal communication among Black female dyads: An assessment of intimacy, gender, and race. Journal of Social Issues, 39, 55-67.

*Smythe, M. J., \& Huddleston, B. (1992). Competition and collaboration: Male and female communication patterns during dyadic interactions. In L. A. M. Perry, L. H. Turner, \& H. M. Sterk (Eds.), Constructing and reconstructing gender: The links among communication, gender, and language (pp. 251-260). Albany: State University of New York Press.

*Snodgrass, S. E. (1985). [Smiling in dyadic interaction as a function of gender and leader/subordinate role]. Unpublished raw data.

Stearns, P. (1994). American cool: Constructing a twentieth-century emotional style. New York: New York University Press.

*Stimel, C. (1982). Self presentation in order to attract a dating partner. Unpublished doctoral dissertation, Ohio State University, Columbus.

*Taylor, D. E., \& Brownlow, S. (1994). Gender differences in language demeanor during television interviews. Unpublished manuscript.

Taylor, S. E., Klein, L. C., Lewis, B. P., Gruenewald, T. L., Gurung, R. A. R., \& Updegraff, J. A. (2000). Biobehavioral responses to stress in females: Tend-and-befriend, not fight-or-flight. Psychological Review, 107, 411-429.

Thorne, B., \& Henley, N. M. (1975). She said/he said: An annotated bibliography of sex difference in language, speech, and nonverbal communication. Pittsburgh, PA: Know.

Tickle-Degnen, L., Hecht, M. A., Harrigan, J. A., Ambady, N., \& Rosenthal, R. (1997). The effect of nonverbal behavior on favorability of impressions formed: Eight meta-analyses. Manuscript in preparation.

*Tidd, K. L., \& Lockard, J. S. (1978). Monetary significance of the affiliative smile: A case for reciprocal altruism. Bulletin of the Psychonomic Society, 11, 344-346.

*Tofalo, R. (1975). Body, head and limb cues, physical distance, and sex of dyad as factors in the nonverbal communication of a disposition to form or avoid a friendship. Unpublished doctoral dissertation, Howard University, Washington, DC.

Turner, J. C., Hogg, M. A., Oakes, P. J., Reicher, S. D., \& Wetherell, M. S. (1987). Rediscovering the social group: A self-categorization theory. Oxford, England: Basil Blackwell.

*Van Vianen, A. E., \& van Schie, E. C. M. (1995). Assessment of male and female behaviour in the employment interview. Journal of Community \& Applied Social Psychology, 5, 243-257.

*Veno, A. E. (1976). Response to approach: A preliminary process oriented study of human spacing. Social Science Information, 15, 93-115.

Von Baeyer, C. L., Sherk, D. L., \& Zanna, M. P. (1981). Impression management in the job interview: When the female applicant meets the male (chauvinist) interviewer. Personality and Social Psychology Bulletin, 7, 45-51.

*Vrij, A., Edward, K., Roberts, K. P., \& Bull, R. (2000). Detecting deceit via analysis of verbal and nonverbal behavior. Journal of Nonverbal Behavior, 24, 239-265.

*Wada, M. (1989). Effects of interpersonal relationship, interpersonal distance, and gender on nonverbal behavior in dyads of strangers and acquaintances. Japanese Journal of Psychology, 60, 31-37.

*Wada, M. (1990). The effect of interpersonal distance change on nonverbal behaviors: Mediating effects of sex and intimacy levels in a dyad. Japanese Psychological Research, 32, 86-96.

Wagner, H. L., Buck, R., \& Winterbotham, M. (1993). Communication of specific emotions: Gender differences in sending accuracy and communication measures. Journal of Nonverbal Behavior, 17, 29-53.

Wagner, H. L., \& Smith, J. (1991). Facial expression in the presence of friends and strangers. Journal of Nonverbal Behavior, 15, 201-214.

Walsh, D. G., \& Hewitt, J. (1985). Giving men the come-on: Effect of eye contact and smiling in a bar environment. Perceptual and Motor Skills, 61, 873-874.

*Waxer, P. H. (1985). Video ethology: Television as a data base for cross-cultural studies in nonverbal displays. Journal of Nonverbal Behavior, 9, 111-120.

*Wellens, A. R., \& Faletti, M. V. (1978). Interrelationships of six measures of interpersonal attraction. Psychological Reports, 42, 1022.

Wierzbicka, A. (1994). Emotion, language and cultural scripts. In S. Kitayama \& H. R. Markus (Eds.), Emotion and culture: Empirical studies of mutual influence (pp. 133-196). Washington, DC: American Psychological Association.

*Willson, A., \& Lloyd, B. (1990). Gender vs. power: Self-posed behavior revisited. Sex Roles, 23, 91-98.

*Wolfe, C. (1971). Human smiling behavior: Sex-linked? Unpublished manuscript, University of Chicago.

Wood, W. (1987). Meta-analytic review of sex differences in group performance. Psychological Bulletin, 102, 53-71.

Wood, W., \& Karten, S. J. (1986). Sex differences in interaction style as a product of perceived sex differences in competence. Journal of Personality and Social Psychology, 50, 341-347.

*Yarmey, A. D. (1989). Recognition memory and expressive behavioral correlates of self as an imaginal prototype in younger and older men and women. Journal of Mental Imagery, 13, 161-170.

*Yarmey, A. D., \& Johnson, J. (1982). Evidence for the self as an imaginal prototype. Journal of Research in Personality, 16, 238-246.

*Yarom, N. (1971). Temporal localization and communication of experimenter expectancy effect with 10-11 year old children. Unpublished doctoral dissertation, University of Illinois, UrbanaChampaign.

Zanna, M. P., \& Pack, S. J. (1975). On the self-fulfilling nature of apparent sex differences in behavior. Journal of Experimental Social Psychology, 11, 583-591.

Zanolli, K., Saudargas, R., \& Twardosz, S. (1990). Two-year olds' re- 
sponses to affectionate and caregiving teacher behavior. Child Study Journal, 20, 35-54.

*Zaslow, M. J., Pedersen, F. A., Suwalsky, J. T. D., \& Rabinovich, B. A. (1989). Maternal employment and parent-infant interaction at one year. Early Child Research Quarterly, 4, 459-478.

*Zebrowitz, L. A., Olson, K., \& Hoffman, K. (1993). Stability of babyfaceness and attractiveness across the life span. Journal of Personality and Social Psychology, 64, 453-466.

*Zuckerman, M., DeFrank, R. S., Hall, J. A., Larrance, D. T., \& Rosenthal, R. (1979). Facial and vocal cues of deception and honesty. Journal of Experimental Social Psychology, 15, 378-396.
Zuckerman, M., \& Driver, R. E. (1985). Telling lies: Verbal and nonverbal correlates of deception. In A. W. Siegman \& S. Feldstein (Eds.), Multichannel integration of nonverbal behavior (pp. 129-147). Hillsdale, NJ: Erlbaum.

Received November 5, 2000 Revision received August 28, 2002 Accepted August 28, 2002

\section{Members of Underrepresented Groups: Reviewers for Journal Manuscripts Wanted}

If you are interested in reviewing manuscripts for APA journals, the APA Publications and Communications Board would like to invite your participation. Manuscript reviewers are vital to the publications process. As a reviewer, you would gain valuable experience in publishing. The $\mathrm{P} \& \mathrm{C}$ Board is particularly interested in encouraging members of underrepresented groups to participate more in this process.

If you are interested in reviewing manuscripts, please write to Demarie Jackson at the address below. Please note the following important points:

- To be selected as a reviewer, you must have published articles in peer-reviewed journals. The experience of publishing provides a reviewer with the basis for preparing a thorough, objective review.

- To be selected, it is critical to be a regular reader of the five to six empirical journals that are most central to the area or journal for which you would like to review. Current knowledge of recently published research provides a reviewer with the knowledge base to evaluate a new submission within the context of existing research.

- To select the appropriate reviewers for each manuscript, the editor needs detailed information. Please include with your letter your vita. In your letter, please identify which APA journal(s) you are interested in, and describe your area of expertise. Be as specific as possible. For example, "social psychology" is not sufficient-you would need to specify "social cognition" or "attitude change" as well.

- Reviewing a manuscript takes time (1-4 hours per manuscript reviewed). If you are selected to review a manuscript, be prepared to invest the necessary time to evaluate the manuscript thoroughly.

Write to Demarie Jackson, Journals Office, American Psychological Association, 750 First Street, NE, Washington, DC 20002-4242. 